\title{
The modelling of intermediate-age stellar populations
}

\section{Average spectra for upper AGB stars, and their use}

\begin{abstract}
A. Lançon and M. Mouhcine ${ }^{1}$
Observatoire Astronomique, Université L. Pasteur \& CNRS: UMR 7550, 11 rue de l'Université, 67000 Strasbourg, France

Received 19 June 2001 / Accepted 15 April 2002

Abstract. The upper Asymptotic Giant Branch (AGB) is populated with oxygen rich and carbon rich Long Period Variables (LPVs). These stars are essential contributors to the near-IR light of intermediate age stellar populations. Individual observed spectra of LPVs are so diverse that they cannot be used directly in the synthesis of galaxy spectra. In this paper, the library of individual spectra of Lançon \& Wood (2000) is used to construct averages that can be incorporated conveniently in population synthesis work. The connection between such spectra and stellar evolution tracks is discussed. In order to select a sorting criterion and to define averaging bins for the LPV spectra, correlations between their spectrophotometric properties are reexamined. While optical properties and broad baseline colours such as $(I-K)$ are well correlated, a large dispersion is observed when these indices are plotted against near-IR ones. This is partly due to the intrinsic width of the upper AGB, which is illustrated by locating each of the multiple observations of individual LPVs on the HR diagram. It is argued that broad baseline colourtemperatures are the most sensible sorting criteria. The properties of the resulting sequence of average spectra indeed vary regularly. We further address: (i) the bolometric corrections and temperature scales needed to associate a spectrum with a given point on a theoretical stellar evolution track (or isochrone), (ii) the simplifying assumptions that will be implicitely made when using the average spectra, (iii) potential biases in the sample of Lançon \& Wood and their effects, (iv) the small contribution of LPVs to the interstellar hydrogen emission lines in galaxies. It is emphasized that an a posteriori calibration of the effective temperature scale remains necessary, until consistent models for the evolution, the pulsation and the spectral appearance of LPVs become available. We suggest a recipe for the use of the average spectra at various metallicities.
\end{abstract}

Key words. stars: AGB and post-AGB - stars: late-type - stars: variable: general - Hertzsprung-Russell diagram infrared: stars - galaxies: stellar content

\section{Introduction}

The prediction of the near-infrared (near-IR) spectra of star clusters and galaxies is subordonate to the existence of stellar spectral libraries with a complete coverage of the evolved stages of stellar evolution. Stellar libraries are also necessary in the construction of near-IR colour-magnitude or twocolour diagrams, which may include narrow-band filter indices or absorption line measurements. A shortcoming of all libraries previously used in population synthesis work (e.g. Kleinmann \& Hall 1986; Terndrup et al. 1991; Lançon \& Rocca-Volmerange 1992; Pickles 1998) is the poor coverage of the upper Asymptotic Giant Branch (AGB). The upper AGB hosts oxygen-rich and carbon-rich Long Period Variables $(\mathrm{LPVs})^{1}$, whose specific spectral features carry the potential of

Send offprint requests to: A. Lançon,

e-mail: lancon@astro.u-strasbg.fr

${ }^{1}$ In agreement with common usage, LPVs include both semiregular and Mira-type variables; Miras are LPVs with large (optical) amplitudes, i.e. $\delta V>2.5$ mag (Kholopov et al. 1985; Lloyd Evans 1983; Hughes \& Wood 1990). revealing the presence of intermediate age populations (Lançon et al. 1999). Indeed, upper AGB stars contribute of the order of $50 \%$ of the $K$ band light over a range of stellar population ages between $\sim 10^{8}$ and $\sim 10^{9}$ years (Persson et al. 1983; Renzini \& Buzzoni 1986; Frogel et al. 1990; Ferraro et al. 1995; Bressan et al. 1998; Girardi \& Bertelli 1998; Lançon 1998; Maraston 1998; Mouhcine \& Lançon 2002).

Recently, Lançon \& Wood (2000; hereafter LW2000) published a library of stellar spectra of luminous cool stars that includes a large sample of instantaneous observations of LPVs. The data span the wavelength range where most of the light of cool stars is emitted $(0.5$ to $2.5 \mu \mathrm{m})$ with a spectral resolving power of $\sim 1100$ longward of $1 \mu \mathrm{m}$, and, with about 100 spectra, provide the most complete data set available to date.

The range of spectrophotometric properties observed in LPVs is large (LW2000; Alvarez et al. 2000). Even the classification of the empirical spectra has many caveats. Sorting algorithms based on optical or on near-IR criteria, on broad band colours or on spectroscopic properties, on mean or on instantaneous data, often give different results. The most conspicuous features, such as the $\mathrm{H}_{2} \mathrm{O}$ vapour absorption bands around 
$1.4 \mu \mathrm{m}$ and $1.9 \mu \mathrm{m}$ (in oxygen rich LPVs), depend on details of the atmospheric structure that change from one pulsation cycle to the next. Such changes will be smoothed out in the integrated light of (large) stellar populations.

The purpose of the present paper is to provide a library of mean stellar spectra of luminous cool variable stars, suitable for direct use in combination with a population synthesis model. The library complements more readily available data for static red giants or supergiants. Both oxygen rich and carbon rich AGB stars are considered. We attempt to thoroughly discuss the impact of technical/physical choices that have to be made at various steps in the preparation and the use of the averaged spectra.

The construction of a library of mean spectra requires the preliminary construction of suitable averaging bins. In principle, one would like to compute energy weighted mean spectra for individual stars, based on spectra taken at various phases over several pulsation cycles and on the light curve. However, this requires an enormous amount of spectroscopic and photometric data. Even the LW2000 data are not sufficient. This paper suggests that colour temperature is the best single parameter to order LPV spectra. The sample of useful input spectra is briefly described in Sect. 2, where the main selection criteria (and thus potential selection biases) are also recalled. Sequences of average spectra are presented in Sect. 3. The statistical properties of the data that justify our selected sorting criteria are also presented in that section.

The discussion of the uncertainties in population synthesis predictions inherent to the chosen averaging procedure must be based on the understanding of two processes that tend to disperse the stars of even a coeval population over quite a broad area of the Hertzsprung-Russell (HR) diagram: the TP-AGB thermal pulse cycles and the LPV pulsation cycles. The theoretical stellar evolution tracks used in population synthesis calculations provide the time evolution of the position of a star in the HR diagram. The thermal pulses are now commonly accounted for in the tracks, but the effects of pulsation, on timescales of hundreds or thousands of days, are not included. One may think of these tracks as providing the evolution of the static parent stars of LPVs. Unfortunately, building non-linear pulsation models and theoretical spectra for LPVs is extremely complex, and current models cannot yet provide the fundamental parameters of the parent star on the basis of one or several instantaneous empirical spectra or colours.

How, then, should one connect the averaged spectra of the new library with locations along the evolutionary tracks? Practical aspects of this question are addressed in Sect. 4. The subsequent discussion addresses fundamental difficulties. In particular, it emphasizes the need for a posteriori calibration of the relation between colour temperatures and effective temperatures (i.e. the temperature scale of the spectra). Potential selection biases in the LW2000 data, and their effect, are also discussed in Sect. 5. Recipes for extensions of the population synthesis calculations to other metallicities than quasi-solar are provided in Sect. 6. A concluding summary is given in Sect. 7. $\mathrm{CDS}^{2}$.

The averaged spectra are available in digital form through

\section{The sample}

The library of LW2000 contains more than 100 merged optical/near-IR spectra. Of these, about one quarter belong to giants of the first giant branch, red supergiants, Galactic Bulge LPVs or luminous red stars of the Large and Small Magellanic Clouds. The remaining spectra are those of pulsating giants in the field of the Milky Way. These are the data we focus on.

The sample of LW2000 was selected to provide a relatively uniform coverage of the period-amplitude plane of LPVs (their Fig. 1). The O-rich LPVs with $V$ band amplitudes $(\delta V)$ smaller than $0.7 \mathrm{mag}$ in the sample show no obvious difference with static giants. We exclude these objects from the subsample used here. The resulting set contains 63 O-rich spectra of LPVs with $\delta V \geq 0.8 \mathrm{mag}$.

The sample of C-rich stars contains only 6 objects, of which one is an S/C star (3 of 21 spectra) and one is a far-IR source (R Lep = IRAS 04573-1452; 3 spectra). Although their amplitudes are larger than 1.5 mag in $V$, the spectra show relatively little variation with phase. The largest amplitude amounts $\delta V \simeq 6 \mathrm{mag}$ for the far-IR source. The next largest amplitude is $\delta V \simeq 3.7$. The spectrophotometric properties of the $\mathrm{C}$ stars in the sample are much less dispersed than those of the O-rich LPVs.

\section{Average spectra for LPVs}

In principle, the ideal spectral library for population synthesis purposes should consist of a large collection of light-curve weighted spectra of individual stars observed photometrically and spectroscopically over several pulsation cycles. There are two main reasons why this is not practical. First, the amount of data required is enormous. In the sample of LW2000, only a few light curves have been sampled at more than 4 points. The actual light curves and phases are generally not accurately known. No more complete empirical spectral library for LPVs is available in the literature. Second, uncertainties in current models make it impossible to determine the physical nature of one particular star (initial mass, current evolutionary status), even when many spectra are available for it. Attempts to follow the "ideal" route fail at a very basic level: the mean spectra of individual stars cannot be sorted into a sequence that behaves regularly enough for practical use.

Along the TP-AGB, the effective temperature globally decreases while the luminosity increases. In this section, we show that the use of effective temperature indicators as basic sorting criteria produces a sequence of O-rich spectra with regularly evolving properties. The tightness of this empirical sequence tells us that surface temperature is indeed a good parameter for the first order classification of the spectra. Other sorting parameters are discussed but are found to be less appropriate. Using the suggested temperature sequence is, in our opinion, the most

\footnotetext{
2 Centre de Données Astrophysiques de Strasbourg, http://cdsweb.u-strasbg.fr/cgi-bin/qcat?]/A+A/391/167, VizieR service.
} 
sensible way to include O-rich LPVs in evolutionary synthesis models for galaxies.

In the case of $\mathrm{C}$-rich stars, both a temperature sequence and a sequence of $\mathrm{C} / \mathrm{O}$ abundance ratio are considered.

\subsection{Oxygen-rich LPVs}

\subsubsection{Temperature sequence}

The spectra of oxygen-rich LPVs selected in Sect. 2 cover a range of $8 \mathrm{mag}$ in $(V-K)$ and $4 \mathrm{mag}$ in $(V-I)$ or $(I-K)^{3}$. These long baseline colours are expected to correlate with effective temperature (Bessell et al. 1989b, 1998), although the absolute calibration of the relation is poorly known for variable stars, and is metallicity-dependent (cf. Sect. 6).

Figure 1 confirms that tight correlations exist between various commonly used photometric temperature indicators. The small dispersion in panels b) and c), which relate $(V-K)$ and $(R-K)$ to $(I-K)$, shows that the three long baseline broad band colours contain equivalent information. In the following, $(I-K)$ is adopted as the primary temperature index. The choice between $(I-K)$ and $(R-K)$ is arbitrary, while $(V-K)$ was rejected because the passbands of standard V filters extend significantly over the edge of the LW2000 data, requiring extrapolation of the data or (as was done here) a truncation of the filter passband.

The VO index of panel f) measures the depth of the VO A$\mathrm{X}(\Delta v=0)$ band at $1.05 \mu \mathrm{m}$, as done by Bessell et al. (1989b) and Alvarez et al. (2000). The absence of the molecular band in the warmer LPV spectra restricts its use as a temperature indicator. $S_{1 / 3}$ and $S_{2 / 3}$ in panels d) and e) are the optical narrow band filter indices defined by Fluks et al. (1994) ${ }^{4}$. For static giants, they are good indicators of the spectral type, although the molecular bands that determine $S_{1 / 3}$ saturate for the coolest stars (spectral types later than M6). The relative dispersion in the figures involving narrow bands is due to the sensitivity of individual molecular bands to the structure of the outer atmosphere, which is affected by details of pulsation. This sensitivity has been discussed by Bessell et al. (1989b) on the basis of Mira models and is found empirically in measured variations of the bands relative to each other or to the colours, from cycle to cycle and with phase (Spinrad \& Wing 1969; Alvarez \& Plez 1998 LW2000). It strengthens the preference given to $(I-K)$ as a temperature indicator.

$(I-K)$ was used to sort the 63 individual spectra into 9 bins of 7 spectra each, as listed in Table 1 and illustrated in Fig. 1a. As a result of this choice, bin sizes in terms of $(I-K)$ are not constant. No binning choice guarantees constant bin sizes in all colours, and choosing constant bin sizes in $(I-K)$ rather than a constant number of spectra per bin would have given $(I-K)$

\footnotetext{
${ }^{3}$ Unless otherwise stated, broad band colours are measured on the reduced spectra using the transmission curves of Bessell (1990) for $V$, $R$ and $I$ (Cousins system), and of Bessell \& Brett (1988) for $J, H$ and $K$ (Johnson-Glass system). Zero colours are adopted for Vega, based on the Vega model spectrum also used by Bessell \& Brett (we thank M. Bessell for providing the latter).

${ }^{4}$ Note that integrated flux ratios are used in that definition, instead of the usual flux density ratios.
}
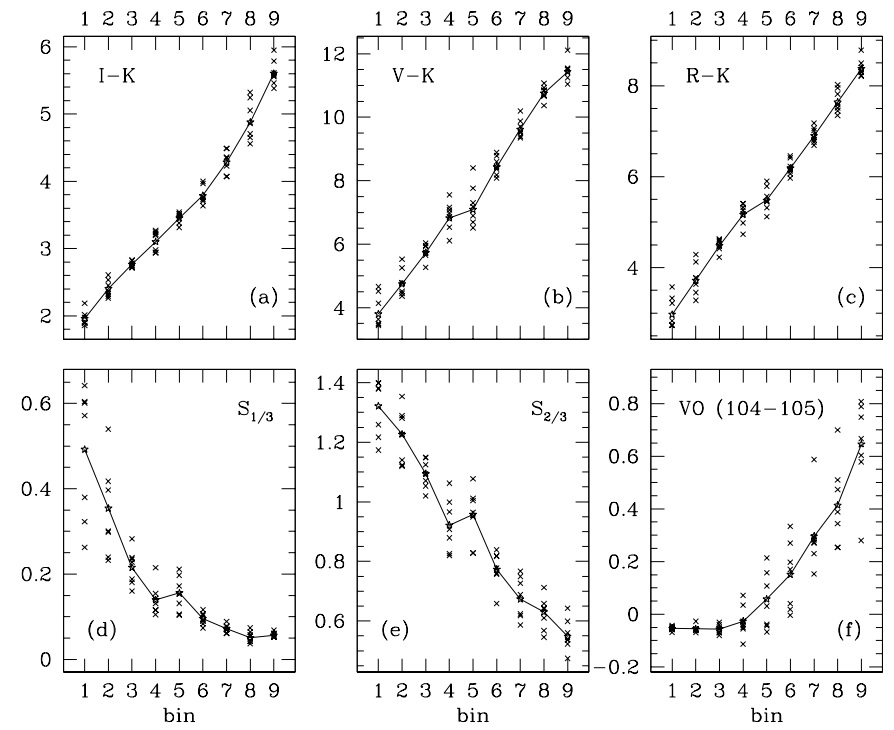

Fig. 1. Temperature sensitive indices as a function of the bin number based on $(I-K)$. The indices of the O-rich LPVs of LW2000 are shown as crosses; the solid lines connect those of the averaged spectra. All colours are in magnitudes, with a value of 0 for Vega. In b), $V$ is measured through a standard filter truncated at $5100 \AA$ (cf. Alvarez et al. 2000).

more importance than it deserves compared to the other colours of Fig. 1. Within each bin, the spectra were normalised to a common mean flux over the whole wavelength range of the data, and were then averaged without further weighting. The resulting sequence of average spectra is displayed in Fig. 2. The maximum of the energy distribution is seen to move from about $7500 \AA$ for the hottest spectrum to about $1.3 \mu \mathrm{m}$ for the coolest. Molecular bands grow more numerous and deeper with decreasing colour temperature. The corresponding photometric index measurements are located on the solid lines in Fig. 1.

\subsubsection{Near-IR properties}

The spectral features of highest relevance to extragalactic studies are located longward of $1 \mu \mathrm{m}$, i.e. at wavelengths where the relative contribution of the AGB stars to the integrated light can be significant. The VO band of Fig. $1 \mathrm{f}$ is one example. The behaviour of the near-IR broad band colours $(J-K),(H-K)$ and $(J-H)$ and of the $\mathrm{CO}$ and $\mathrm{H}_{2} \mathrm{O}$ absorption bands is shown in Fig. 3. All these indices are based on the Hubble Space Telescope NICMOS filter passbands ${ }^{5}$. They are given in magnitudes, with the assumption that all colours are 0 for Vega. The solid lines refer to the properties of our prefered sequence of averages, described in Sect 3.1.1. The dashed and dot-dashed lines are obtained respectively with 7 bins of 9 spectra each, and with 10 bins of 6 or 7 spectra each.

Figure 3 shows that the near-IR broad band colours $(J-K),(H-K)$ and $(J-H)$ correlate with the $(I-K)$

\footnotetext{
${ }^{5}$ Many molecular index definitions are found in the literature. We recommend that users measure the index of their choice on the actual spectra instead of attempting a conversion from the plots in this section.
} 
Table 1. Contents of the O-rich temperature bins.

\begin{tabular}{|c|c|c|c|c|c|c|}
\hline $\begin{array}{l}\text { Bin } \\
\text { (1) }\end{array}$ & $\begin{array}{r}\text { Name } \\
\text { (2) }\end{array}$ & $\begin{array}{l}\text { Date } \\
\text { (3) }\end{array}$ & $\begin{array}{l}\text { Type } \\
\text { (4) }\end{array}$ & $\begin{array}{c}\text { Period } \\
\text { (5) }\end{array}$ & $\begin{array}{l}\bar{~} \overline{\delta V} \\
(6)\end{array}$ & $\begin{array}{c}(I-K) \\
(7)\end{array}$ \\
\hline 1 & TCen & Jul. 96 & $\mathrm{Sa}$ & 90.4 & 3.5 & 1.86 \\
\hline 1 & TCen & Jan. 96 & $\mathrm{Sa}$ & 90.4 & 3.5 & 1.90 \\
\hline 1 & BD Hya & Jul. 96 & $\mathrm{Sa}$ & 117.4 & 1.8 & 1.90 \\
\hline 1 & S Car & Jun. 95 & M & 149.5 & 5.4 & 1.90 \\
\hline 1 & S Car & Dec. 95 & M & 149.5 & 5.4 & 1.98 \\
\hline 1 & TCen & Mar. 96 & $\mathrm{Sa}$ & 90.4 & 3.5 & 2.01 \\
\hline 1 & RS Hor & Mar. 96 & $\mathrm{M}$ & 202.9 & 5.2 & 2.19 \\
\hline 2 & S Lib & May 96 & M & 192.9 & 5.5 & 2.26 \\
\hline 2 & S Lib & Jun. 95 & M & 192.9 & 5.5 & 2.29 \\
\hline 2 & S Car & May 96 & M & 149.5 & 5.4 & 2.32 \\
\hline 2 & TCen & May 96 & $\mathrm{Sa}$ & 90.4 & 3.5 & 2.36 \\
\hline 2 & $\mathrm{UCrt}$ & Jan. 96 & M & 169. & 4. & 2.45 \\
\hline 2 & BD Hya & May 96 & $\mathrm{Sa}$ & 117.4 & 1.8 & 2.53 \\
\hline 2 & BD Hya & Jan. 96 & $\mathrm{Sa}$ & 117.4 & 1.8 & 2.61 \\
\hline 3 & S Car & Jan. 96 & M & 149.5 & 5.4 & 2.71 \\
\hline 3 & R Phe & May 96 & M & 269.3 & 6.9 & 2.72 \\
\hline 3 & S Car & Jul. 96 & M & 149.5 & 5.4 & 2.73 \\
\hline 3 & S Phe & Dec. 95 & $\mathrm{Sb}$ & 141. & 2. & 2.75 \\
\hline 3 & UZ Hya & Jan. 96 & M & 261. & 5.7 & 2.78 \\
\hline 3 & UZ Hya & Mar. 96 & M & 261. & 5.7 & 2.82 \\
\hline 3 & R Phe & Jul. 96 & M & 269.3 & 6.9 & 2.83 \\
\hline 4 & U Crt & May 96 & M & 169. & 4. & 2.93 \\
\hline 4 & KV Car & May 96 & $\mathrm{Sb}$ & 150. & 0.8 & 2.94 \\
\hline 4 & RS Hor & Jan. 96 & M & 202.9 & 5.2 & 2.98 \\
\hline 4 & RS Hya & Jun. 95 & M & 338.6 & 5.2 & 3.19 \\
\hline 4 & RS Hya & May 96 & M & 338.6 & 5.2 & 3.22 \\
\hline 4 & S Phe & Jan. 96 & $\mathrm{Sb}$ & 141. & 2. & 3.24 \\
\hline 4 & SY Vel & Mar. 96 & $\mathrm{Sb}$ & 63. & 1.3 & 3.27 \\
\hline 5 & KV Car & Jan. 96 & $\mathrm{Sb}$ & 150. & 0.8 & 3.31 \\
\hline 5 & S Car & Mar. 96 & M & 149.5 & 5.4 & 3.38 \\
\hline 5 & SY Vel & Jan. 96 & $\mathrm{Sb}$ & 63. & 1.3 & 3.45 \\
\hline
\end{tabular}

colour-temperature, but with a large dispersion and a relatively shallow slope. If $(I-K)$ really is a good effective temperature estimator for O-rich LPVs, then it is clear that the near-IR colours are poor ones. Bins based on near-IR colours would mix spectra with very diverse general energy distributions, optical spectra and spectral types.

The observed decrease in the near-IR colour indices between $(I-K)=3.3$ and $(I-K)=4.5$ (bins 5 to 7 ) is the result of the competition between the influences of temperature and of molecular absorption. This decrease is statistically significant in our sample, in particular in $(J-H)$ : the size of the step is about 3 times the estimated rms error on the mean colour (rms dispersion of the data divided by $\sqrt{7}$, as we have 7 spectra per bin). However, it is unclear whether it would be seen in larger samples. In addition, the step is relatively small when compared to the whole range of the data, and it is comparable to typical observational uncertainties in near-IR photometric observations. Our summary of these data is that the mean $(J-K),(H-K)$ and $(J-H)$ of O-rich LPVs are approximately constant between $(I-K) \simeq 3$ and $(I-K) \simeq 5$ (i.e. bins $\sim 3$ to $\sim 8$ ).

Spectra with similar energy distributions display an impressive variety of near-IR molecular absorption spectra, as

\begin{tabular}{crllccc}
\hline \hline Bin & Name & Date & Type & Period & $\delta V$ & $(I-K)$ \\
$(1)$ & $(2)$ & $(3)$ & $(4)$ & $(5)$ & $(6)$ & $(7)$ \\
\hline 5 & S Phe & Jul.96 & Sb & 141. & 2. & 3.49 \\
5 & S Phe & May 96 & Sb & 141. & 2. & 3.51 \\
5 & RY Cra & Jun.95 & M & 195. & 1.9 & 3.51 \\
5 & RS Hya & Jul.96 & M & 338.6 & 5.2 & 3.54 \\
6 & RS Lib & May 96 & M & 217.6 & 6. & 3.63 \\
6 & RS Hya & Apr.96 & M & 338.6 & 5.2 & 3.70 \\
6 & RZ Car & May 96 & M & 272.8 & 6.2 & 3.73 \\
6 & SV Tel & Jun.95 & M & 225.5 & 3. & 3.76 \\
6 & R Phe & Dec.95 & M & 269.3 & 6.9 & 3.77 \\
6 & RS Hor & Dec.95 & M & 202.9 & 5.2 & 3.97 \\
6 & R Cha & Dec.95 & M & 334.6 & 6.7 & 4.00 \\
7 & RS Hya & Mar.96 & M & 338.6 & 5.2 & 4.07 \\
7 & X Men & Jul.96 & M & 380. & 3.6 & 4.07 \\
7 & R Phe & Jan.96 & M & 269.3 & 6.9 & 4.23 \\
7 & R Cha & May 96 & M & 334.6 & 6.7 & 4.34 \\
7 & WW Sco & May 96 & M & 431. & 4.1 & 4.36 \\
7 & RZCar & Jun.95 & M & 272.8 & 6.2 & 4.49 \\
7 & RZCar & Jul.96 & M & 272.8 & 6.2 & 4.49 \\
8 & SV Lib & Jul.96 & M & 402.7 & 1.3 & 4.56 \\
8 & SV Lib & Jun.95 & M & 402.7 & 1.3 & 4.65 \\
8 & SV Lib & May96 & M & 402.7 & 1.3 & 4.70 \\
8 & R Cha & Jan.96 & M & 334.6 & 6.7 & 4.86 \\
8 & CM Car & Jan.96 & M & 335. & 2.5 & 5.05 \\
8 & RS Hya & Jan.96 & M & 338.6 & 5.2 & 5.24 \\
8 & X Men & Dec.95 & M & 380. & 3.6 & 5.32 \\
9 & RZCar & Mar.96 & M & 272.8 & 6.2 & 5.38 \\
9 & X Men & Mar.96 & M & 380. & 3.6 & 5.46 \\
9 & X Men & Jan.96 & M & 380. & 3.6 & 5.55 \\
9 & RZ Car & Jan.96 & M & 272.8 & 6.2 & 5.57 \\
9 & WW Sco & Jun.95 & M & 431. & 4.1 & 5.61 \\
9 & RS Lib & Jun.95 & M & 217.6 & 6. & 5.79 \\
9 & R Cha & Mar.96 & M & 334.6 & 6.7 & 5.95 \\
\hline
\end{tabular}

illustrated in Fig. 4. Again, this shows how sensitive the spectral signatures of molecules such as $\mathrm{H}_{2} \mathrm{O}$, that form far in the outer atmosphere (Matsuura et al. 2001, models of Hofmann et al. 1998: priv. communication by M. Scholz), are to the instantaneous pulsation-determined structure of the latter. We will attempt to further interpret these differences in Sect. 3.1.3. Differences in the width and depth of the wings of the broad $\mathrm{H}_{2} \mathrm{O}$ absorption bands contribute significantly to the spread around the mean near-IR broad band colours. CO absorption in the $H$ band plays a smaller role. Real deviations in the pseudo-continuum between 1 and $1.3 \mu \mathrm{m}$ occur (see Sect. 3.5 of LW2000) and also contribute to the spread.

Along the sequence of averaged spectra, the water vapour absorption bands deepen and widen with decreasing temperature. The measurements shown are based on the HST/NICMOS filters F204M and F222M:

$$
\mathrm{H}_{2} \mathrm{O}=-2.5 \log \left[F_{\lambda}(\mathrm{F} 204 \mathrm{M}) / F_{\lambda}(\mathrm{F} 222 \mathrm{M})\right]-\mathrm{H}_{2} \mathrm{O}(\text { Vega }) .
$$

The first is centered at $2.04 \mu \mathrm{m}$, deep in one of the $\mathrm{H}_{2} \mathrm{O}$ bands, the second at $2.22 \mu \mathrm{m}$, at a quasi-continuum point. The lower envelope of the data points in Fig. 3d illustrates the mere effect of the change in colour-temperature for stars with shallow or inexistent $\mathrm{H}_{2} \mathrm{O}$ bands. At a given $(I-K)$, star-to-star differences 


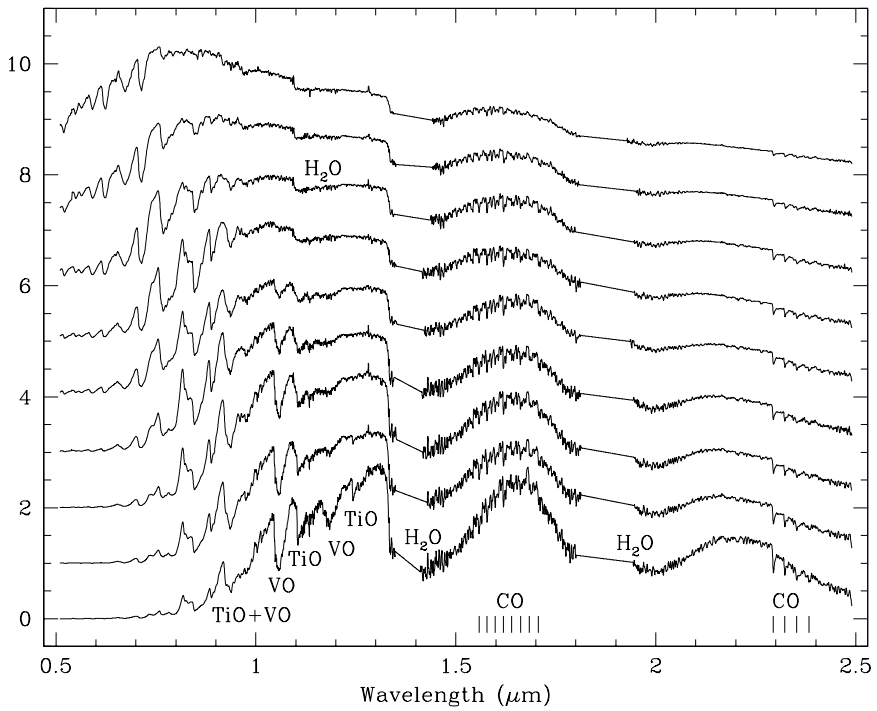

Fig. 2. The sequence of O-rich LPV spectra, obtained after sorting the spectra according to $(I-K)$. Linear flux density units are used $\left(F_{\lambda}\right)$. The spectra have been normalized to a common integrated flux, and each is offset from the lower one by one unit. Straight segments interpolate through the regions where the telluric absorption was too strong for a satisfactory correction.
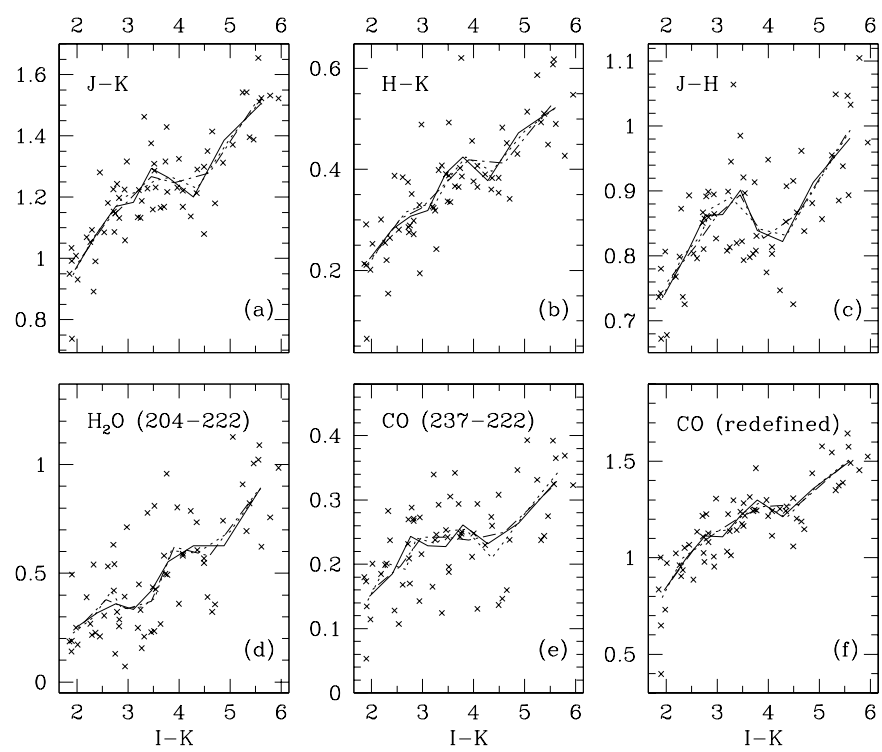

Fig. 3. Common near-IR indices as a function of $(I-K)$, for O-rich LPVs. Lines show the properties of averaged spectra, with the three bin definitions given in the text. Plots d) and e) are flux density ratios, measured through the bandpasses of the HST/NICMOS/Camera 2 filters F204M, F222M, F237M, as labeled, and converted to magnitudes (0 for Vega). The CO index in plot f) is colour-corrected (see text).

of up to $0.7 \mathrm{mag}$ are seen in the (204-222) index. Figure 4 shows that similar spreads are expected in other band wings of this molecule, thus affecting in particular the broad band photometry. For instance, the star with the largest $(H-K)$ (at $(I-K) \simeq 3.8$ ) also has extreme water bands (second spectrum from the top of Fig. 4).

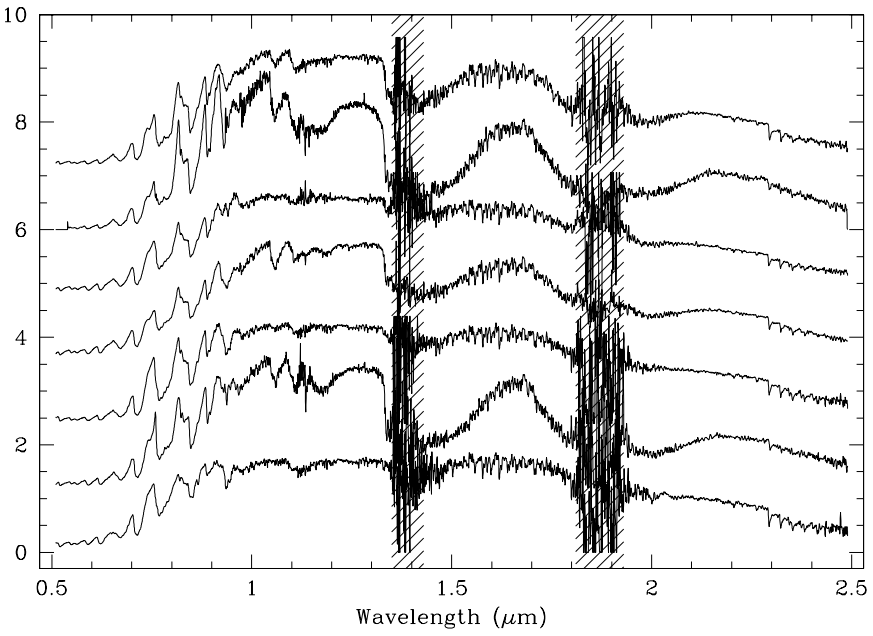

Fig. 4. The seven O-rich LPVs of bin number 5 (in the order of Table 1 from the bottom upwards). An illustration of the variety of near-IR properties seen at a given $(I-K)$.

The $\mathrm{CO}$ index in Fig. 3e measures the ratio of the flux densities at $2.37 \mu \mathrm{m}$ and $2.22 \mu \mathrm{m}$, based on the HST/NICMOS filters F222M and F237M:

$$
\mathrm{CO}=-2.5 \log \left[F_{\lambda}(\mathrm{F} 237 \mathrm{M}) / F_{\lambda}(\mathrm{F} 222 \mathrm{M})\right]-\mathrm{CO}(\text { Vega }) .
$$

The correlation is poor, because stronger $\mathrm{CO}$ band strengths occur in redder stars and these continuum changes nearly compensate for the increasing molecular absorption. The index shown in Fig. 3f includes a colour correction: in the above formula, $F_{\lambda}(\mathrm{F} 237 \mathrm{M})$ is replaced by the linear extrapolation to $2.37 \mu \mathrm{m}$ of the flux densities measured in the filters F165M $(1.65 \mu \mathrm{m})$ and F222M $(2.22 \mu \mathrm{m})$. With this redefinition, the flux in the CO band is compared to an estimate of the continuum flux at the same wavelength, rather than to a measurement in an offset continuum filter. A strong correlation with $(I-K)$ becomes apparent. In fact, the redefined $\mathrm{CO}$ index combines the information contained in the standard $\mathrm{CO}$ index with the information contained in the colour $(H-K)$, providing a better correlation with $(I-K)$ than any of these two indices separately (the new linear correlation coefficient is 0.82 ; it was 0.57 for the standard $\mathrm{CO}$ index and 0.77 for $H-K$ ).

For comparison, we note that the LW2000 sample contains static giants with $(I-K)$ in the range $2-3.1$ and supergiants in the range 2.7-4.5. For all these objects, the $\mathrm{H}_{2} \mathrm{O}$ index of Fig. $3 \mathrm{~d}$ remains below 0.29 . The $\mathrm{CO}$ indices of giants lie along the mean LPV lines in Figs. 3e and f, while those of supergiants lie dispersed along the upper envelope of the LPV distributions.

In view of the large spread of near-IR properties found among O-rich LPVs, it came as a surprise that the sequence of average spectra described in Sect. 3.1.1 displayed such a regular behaviour. Regularity is essential for population synthesis applications. The effects of pulsation on the upper atmospheres of LPVs are responsible for a large scatter in the properties of individual spectra, but do not completely wash out the underlying global effect of temperature. The sample of LW2000, for the first time, provides enough data to recover these trends. 


\subsubsection{Secondary parameters for O-rich LPV spectra}

As temperature decreases along the TP-AGB, luminosities rise, radii increase, and masses decrease due to mass loss. As a consequence, stellar pulsation properties change. Because of the effects of initial mass, of thermal pulses and of LPV pulsation, stars with different pulsation properties can be found at a given TP-AGB temperature. Essential near-IR features such as the $\mathrm{H}_{2} \mathrm{O}$ bands depend on pulsation, even though they also correlate with effective temperature. In evolving stellar populations, the average luminosity of TP-AGB stars decreases with age and a decrease of the average period is thus expected from empirical period-luminosity correlations (e.g. Feast et al. 1989; Wood 1990; Wood et al. 1999). This example illustrates that direct effects of pulsation properties on spectra at a given temperature will have consequences on population synthesis predictions. In principle, population synthesis models should include a gradual evolution of the pulsation properties instead of simply considering a dichotomy between variable and non-variable TP-AGB stars.

We have investigated whether empirical correlations in the LW2000 data between spectrophotmetric properties and pulsation characteristics justify the use of pulsation criteria rather than temperature in the definition of averaging bins. Correlations would have to be significantly better than those of Fig. 3, to compensate for the fact the theoretical predictions for the evolution of period and amplitude along the TP-AGB (or their empirical determination) are even more uncertain than temperature predictions and are not included in current population synthesis codes. As the conclusion is negative, we only summarize the main empirical results here.

In Fig. 5, selected indices relevant to stellar population studies are plotted against period. Correlations are clearly present in all panels. The molecular indices show a larger dispersion at a given period than they do within the colourtemperature bins of Fig. 3. The spread in $(J-H)$ and $(H-K)$ versus period is comparable to the spread seen in Fig. 3. We note that no systematic correlations are found between the residuals in Fig. 3 and period: for a given spectrophotometric index, the value and sign of the correlation coefficient changes randomly from one temperature bin to the next. This probably results from poor sampling of intrinsically dispersed relations.

Figure 6 is the equivalent of Fig. 5 for amplitude. Correlations between amplitude and spectrophotometric indices in the sample have a low level of significance, except for $\mathrm{H}_{2} \mathrm{O}$ (the correlation coefficient is 0.3 , which for 63 data points is significant at the $98 \%$ level; other correlation coefficients are below 0.15). Both strong and weak molecular features are found at all amplitudes. We recall however that the smallest amplitude in the sample is of $0.8 \mathrm{mag}$ and that no strong bands of water vapour or VO are seen in the spectra of the static or quasi-static giant star spectra of LW2000. Again, no systematic correlations were found between the residuals of the relations of Fig. 3 and pulsation amplitude.

With the available sample, the selection of temperature as a primary sorting criterion for the spectra remains the most sensible choice. More stellar data are necessary to correctly define
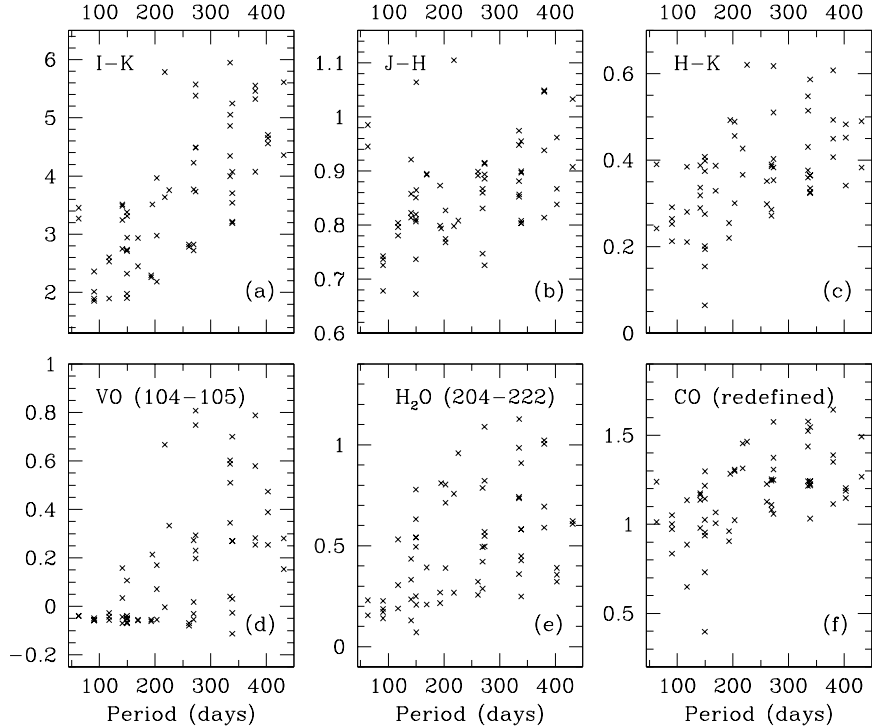

Fig. 5. Colours and near-IR molecular indices versus pulsation period, for O-rich LPVs.
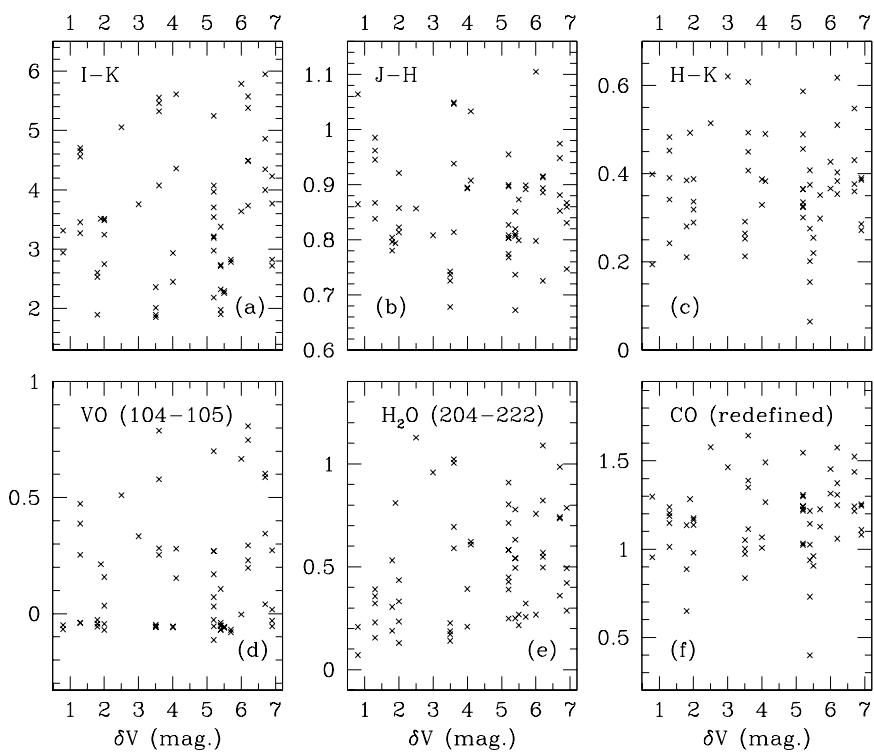

Fig. 6. Colours and near-IR molecular indices versus optical pulsation amplitude, for O-rich LPVs.

the relations between pulsation properties and spectra within individual temperature bins.

\subsection{Carbon-rich LPVs}

Depending on initial mass and metallicity, some TP-AGB stars will become carbon stars through convective dredge-up of freshly synthesized carbon from the core, induced by thermal pulses (e.g. Iben \& Renzini 1983). Carbon stars are essential contributors to the near-IR emission from AGB stars, in particular at subsolar metallicities (Persson et al. 1983; Frogel et al. 1990). The spectral library contains 21 complete spectra of carbon-rich LPVs. On the basis of theoretical spectra, Loidl et al. (2001, hereafter LLJ2001) showed that $(R-J)$ and $(R-H)$ are among the best effective temperature indicators for 
these stars. We will (arbitrarily) use $(R-H)$ here. The far-IR source R Lep is so red that it must be treated separately. R Lep excluded, the range of $(R-H)$ values in the sample does not justify more than two or three averaging bins.

The suggested temperature sequence for C-rich stars contains 3 averages of 6 spectra each (see Table 2), followed by the individual spectrum of R Lep near maximum light, and finally the average of the 3 R Lep spectra near minimum light (the optical spectrum in the 4th bin is an average of bins 3 and 5, because no data was obtained). It is presented in Fig. 7. The averaging procedure is as for O-rich LPVs. The spectrophotometric properties of the data bins are shown in Fig. 8. The $\mathrm{C}_{2}$ index measures the strength of the bandhead at $1.77 \mu \mathrm{m}$. To avoid the spectral region of strong telluric absorption corrections and of contamination by $\mathrm{CN}$, the "continuum" flux density is measured over the narrow range $1.752-1.762 \mu \mathrm{m}$ and the band flux density over 1.768-1.782 $\mu \mathrm{m}$ (Alvarez et al. 2000); the index is given in magnitudes. The models of Gautschy (2001) demonstrate that the $\mathrm{C}_{2}$ bandhead is sensitive to the $\mathrm{C} / \mathrm{O}$ ratio, the ${ }^{12} \mathrm{C} /{ }^{13} \mathrm{C}$ ratio and microturbulence. This contributes to the dispersion in panel e). More data is needed to assess the significance of the trend in panels e) and f).

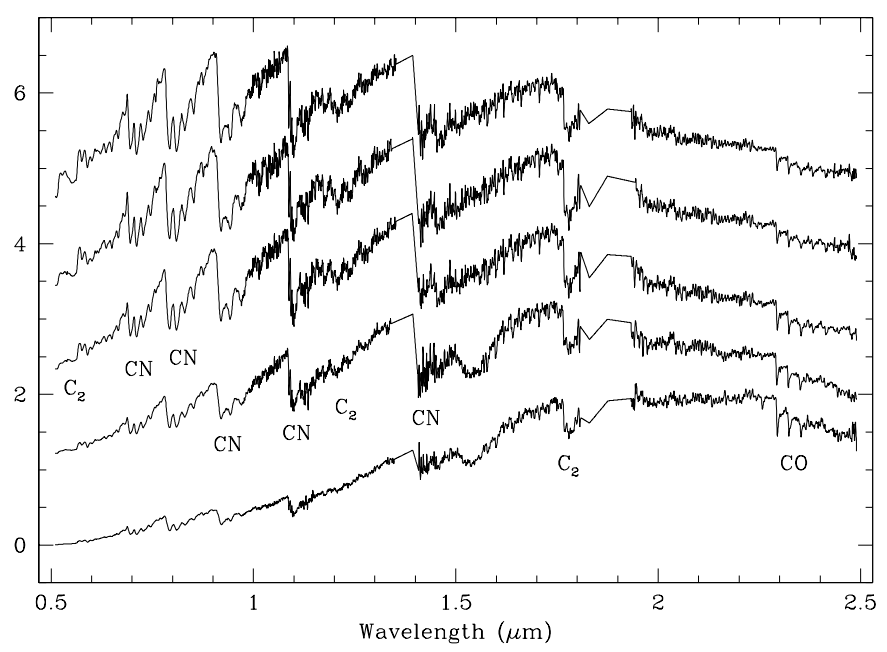

Fig. 7. A temperature sequence of carbon star spectra. Linear flux density units are used $\left(F_{\lambda}\right.$, after normalization to a common total flux, with offsets of 1.1). In the regions irremediably affected by telluric absorption, around 1.35 and $1.9 \mu \mathrm{m}$, we have drawn segments by hand that approximately follow the theoretical shape of molecular bands (LLJ2001). The carrier of the molecular band around $1.55 \mu \mathrm{m}$ is uncertain (Gautschy 2001 suggests a combination of $\mathrm{C}_{2} \mathrm{H}_{2}$ and $\mathrm{HCN}$ ).

Alternative criteria in the construction of averaging bins for carbon stars are $\mathrm{C} / \mathrm{O}$ or ${ }^{12} \mathrm{C} /{ }^{13} \mathrm{C}$, because their effects on the spectral signatures are significant. Both vary along the TP-AGB as a consequence of third dredge-up episodes and envelope burning, in a way that is becoming predictable (Renzini \& Voli 1981; Forestini \& Charbonnel 1997; Marigo 2001). Binning with respect to $\mathrm{C} / \mathrm{O}$ can be done on the basis of estimates of LLJ2001. It is best to isolate the S/C star BHCru in one bin, to combine TCae and RU Pup data, and to group Y Hya and S Cen. Unfortunately, current atmosphere models do not allow us to estimate the ratio for stars as cool as RLep.
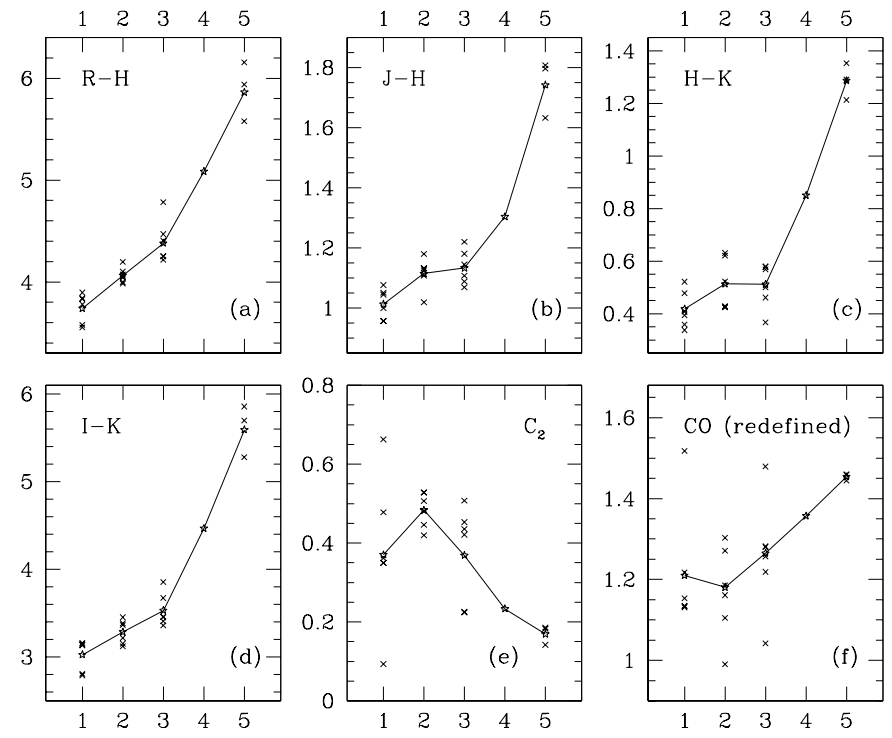

Fig. 8. Spectrophotometric properties of carbon stars as a function of the bin number based on $(R-H)$. The indices for instantaneous spectra are shown as crosses; the solid lines connect those of the averaged spectra (bin 4 contains only 1 spectrum).

The empirical sequence of increasing $\mathrm{C} / \mathrm{O}$ ratios is characterised by increasing $\mathrm{C}_{2}$ band depths (at $1.77 \mu \mathrm{m}$, around $1.2 \mu \mathrm{m}$ and below $5700 \AA$ ), and by decreasing apparent strengths of the $\mathrm{CO}$ bandheads around $1.6 \mu \mathrm{m}$ and $2.3 \mu \mathrm{m}$ as a result of heavier contamination by $\mathrm{CN}$ and $\mathrm{C}_{2}$ (cf. Fig. 4 of LLJ2001). The $\mathrm{C} / \mathrm{O}$ and ${ }^{12} \mathrm{C} /{ }^{13} \mathrm{C}$ sequences based on the LLJ2001 estimates are similar (though in reverse order). We note however that a degeneracy with the effects of turbulent velocity makes ${ }^{12} \mathrm{C} /{ }^{13} \mathrm{C}$ estimates uncertain. The inverse correlation between $\mathrm{C} / \mathrm{O}$ and ${ }^{12} \mathrm{C} /{ }^{13} \mathrm{C}$ found in the LW2000 sample, and values of ${ }^{12} \mathrm{C} /{ }^{13} \mathrm{C}$ as low as 3.5 are not typical from the stellar evolution point of view.

Pulsation has small effects on the spectrum below $2.5 \mu \mathrm{m}$, except for the coolest carbon stars, where it allows a deep band to form around $1.55 \mu \mathrm{m}$ (Fig. 7 and Gautschy 2001).

\subsection{Dust-enshrouded LPVs}

$\mathrm{OH} / \mathrm{IR}$ stars and their carbon rich counterparts are produced on the upper AGB when the dust produced in the heavy winds of an LPV builds a thick enough envelope to hide the central star at optical wavelengths. At a given bolometric luminosity, their contribution to the near-IR light is smaller than for unobscured stars but their mid-IR emission is significant, an effect that may be useful in searches for intermediate age stellar populations (Bressan et al. 1998). More than the effective temperature of the central star, the amount of dust (which is related to the mass loss and, indirectly, also to the effective temperature) determines the near-IR energy output.

To represent the optical and near-IR emission of dustenshrouded sources, we suggest to use reddened versions of the average spectra provided in this paper. Although circumstellar dust might be of a different nature than the average interstellar dust grains, the LW2000 data suggest that the Milky Way extinction law (e.g. Cardelli et al. 1989, with $A v / E(B-V)=3.1$ ) 
Table 2. Contents of the C-rich temperature bins.

\begin{tabular}{|c|c|c|c|c|c|c|}
\hline $\begin{array}{l}\text { Bin } \\
\text { (1) }\end{array}$ & $\begin{array}{r}\text { Name } \\
\text { (2) }\end{array}$ & $\begin{array}{l}\text { Date } \\
\text { (3) }\end{array}$ & $\begin{array}{l}\mathrm{C} / \mathrm{O}^{a} \\
\text { (4) }\end{array}$ & $\begin{array}{c}\text { Period } \\
\text { (5) }\end{array}$ & $\begin{array}{l}\delta V \\
(6)\end{array}$ & $\begin{array}{c}(R-H) \\
\text { (7) }\end{array}$ \\
\hline 1 & $\mathrm{TCae}$ & Dec. 95 & 1.05 & 156. & 1.8 & 3.55 \\
\hline 1 & $\mathrm{TCae}$ & Jan. 96 & 1.05 & 156. & 1.8 & 3.58 \\
\hline 1 & S Cen & Jan. 96 & 1.40 & 65. & 1.5 & 3.78 \\
\hline 1 & $\mathrm{TCae}$ & Mar. 96 & 1.05 & 156. & 1.8 & 3.83 \\
\hline 1 & BHCru & Jan. 96 & 1.01 & 421 & 2.8 & 3.84 \\
\hline 1 & Y Hya & Dec. 95 & 1.40 & 302.8 & 3.7 & 3.89 \\
\hline 2 & RU Pup & Dec. 95 & 1.10 & 425. & 1.9 & 3.98 \\
\hline 2 & RU Pup & Jun. 95 & 1.10 & 425. & 1.9 & 4.00 \\
\hline 2 & Y Hya & May 96 & 1.40 & 302.8 & 3.7 & 4.04 \\
\hline 2 & Y Hya & Jan. 96 & 1.40 & 302.8 & 3.7 & 4.07 \\
\hline 2 & Y Hya & Mar. 96 & 1.40 & 302.8 & 3.7 & 4.10 \\
\hline 2 & Y Нуа & Jul. 96 & 1.40 & 302.8 & 3.7 & 4.20 \\
\hline 3 & RU Pup & Jan. 96 & 1.10 & 425. & 1.9 & 4.22 \\
\hline 3 & RU Pup & Mar. 96 & 1.10 & 425. & 1.9 & 4.25 \\
\hline 3 & Y Нya & Jun. 95 & 1.40 & 302.8 & 3.7 & 4.26 \\
\hline 3 & BHCru & May 96 & 1.01 & 421. & 2.8 & 4.40 \\
\hline 3 & RU Pup & May 96 & 1.10 & 425. & 1.9 & 4.47 \\
\hline 3 & BHCru & May 96 & 1.01 & 421. & 2.8 & 4.78 \\
\hline 4 & R Lep & Nov. 97 & - & 427.1 & 6.2 & 5.08 \\
\hline 5 & R Lep & Dec. 95 & - & 427.1 & 6.2 & 5.58 \\
\hline 5 & R Lep & Jan. 96 & - & 427.1 & 6.2 & 5.94 \\
\hline 5 & R Lep & Mar. 96 & - & 427.1 & 6.2 & 6.16 \\
\hline
\end{tabular}

Note to Table 2:

${ }^{a}$ Atmospheric number ratio of carbon to oxygen, when available, according to the estimates of LLJ2001.

can be used to first order for that purpose. The library of LW2000 contains a handful of spectra of OH/IR sources. In Fig. 9, AFGL1686 and WX Psc are compared with reddened versions of the coolest average spectrum of Sect. 3.1.1, which has similar bands of $\mathrm{H}_{2} \mathrm{O}$, TiO and VO. The agreement is rather good (and even better agreements can be found with reddened versions of selected individual spectra of the initial spectral library). The Milky Way extinction law creates no obvious discrepancy. More data will be needed to investigate this question further, and to perform similar tests with carbon rich stars.

\section{Using the spectra in population synthesis codes}

For practical use in connection with theoretical stellar evolution tracks, the empirical spectra must be properly normalized and a quantitative effective temperature scale must be adopted. These aspects are discussed in Sects. 4.1 and 4.2 below. Of course, the stellar evolution tracks must also provide the transitions between O-rich and C-rich atmospheres, and between optically visible sources and dust-enshrouded infrared sources. The discussion of these aspects of theoretical stellar evolution lies outside the scope of this paper.

\subsection{Bolometric corrections}

Bolometric corrections describe the relation between the spectral flux densities and the bolometric luminosity $L_{\mathrm{bol}}$. As the

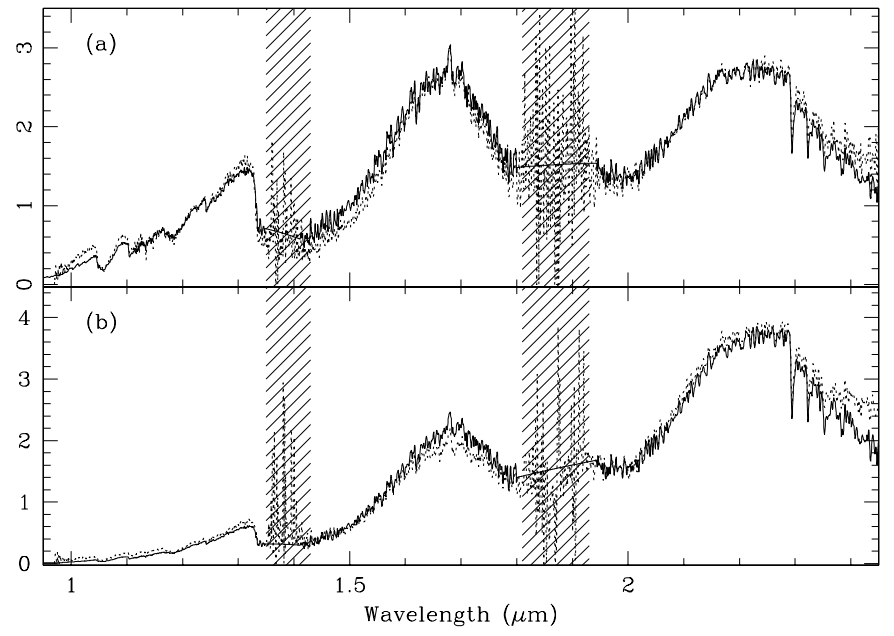

Fig. 9. a) Average of the April 1995 and May 1996 spectra of AFGL $1686=$ IRAS 14068-0730 (dotted) and reddened version of the spectrum of bin 9 of Sect. 3.1.1, using the Milky Way extinction law with $A v=3$ (solid). b) Spectrum of WXPsc=IRAS 01037+1219 (dotted) and reddened spectrum of bin 9 with $A v=18$ (solid). $F_{\lambda}$ units are arbitrary.

LW2000 data encompass most of energy emitted by the LPVs, only small corrections are necessary to convert the measured integrated flux into a bolometric flux. For oxygen rich stars, Alvarez et al. (2000) computed these corrections by extrapolating the LW2000 data with the best-fitting cool M giant models. They found that on average about $20 \%$ of the light is emitted outside the range of the available data. In practice, because blackbodies are poor representations of the infrared emission of cool stars (Fluks et al. 1994; Aringer et al. 1999; Jørgensen et al. 2001), we suggest to simply extrapolate the data linearly to a nil flux at $3500 \AA$ on the blue side and to $50000 \AA$ on the red side. These extrapolation limits are chosen to reproduce the results of Alvarez et al., as shown in Fig. 10a. The long wavelength extrapolation also provides agreement with the integrated fluxes measured longward of $2.5 \mu \mathrm{m}$ for the six ISO satellite spectra of oxygen rich LPVs we have examined ${ }^{6}$, to within random deviations smaller than $10 \%$ (i.e. a few percent on $L_{\text {bol }}$ ).

The infrared emission of carbon stars also differs significantly from a blackbody (e.g. Goebel et al. 1978; Aoki et al. 1999). According to a subset of the models described in LLJ2001, and found to provide satisfactory agreement with ISO data (Jørgensen et al. 2000), the wavelength range of the LW2000 data again comprises $80 \%$ of the flux, and only a few percent of the missed flux comes from $\lambda<5000 \AA$. The total flux of ISO spectra ${ }^{7}$ longward of $2.5 \mu \mathrm{m}$ is equivalent to the suggested linear extrapolation, with object-to-object differences that amount to variations of up to $10 \%$ in the total fluxes. The absence of ISO counterparts for the LW2000 stars

\footnotetext{
${ }^{6}$ SV Peg, T Sge, V1351 Cyg, V584 Apl, VY Cas and CE And; available from the public domain page of Kerschbaum, Loidl, Hron, Kerber, and Rauch: www.astro.univie.ac.at/ fzi/ AGB/agb2pn.html.

7 TX Psc, V460 Cyg and S Cep; retrieved from the Vienna web site mentioned above.
} 

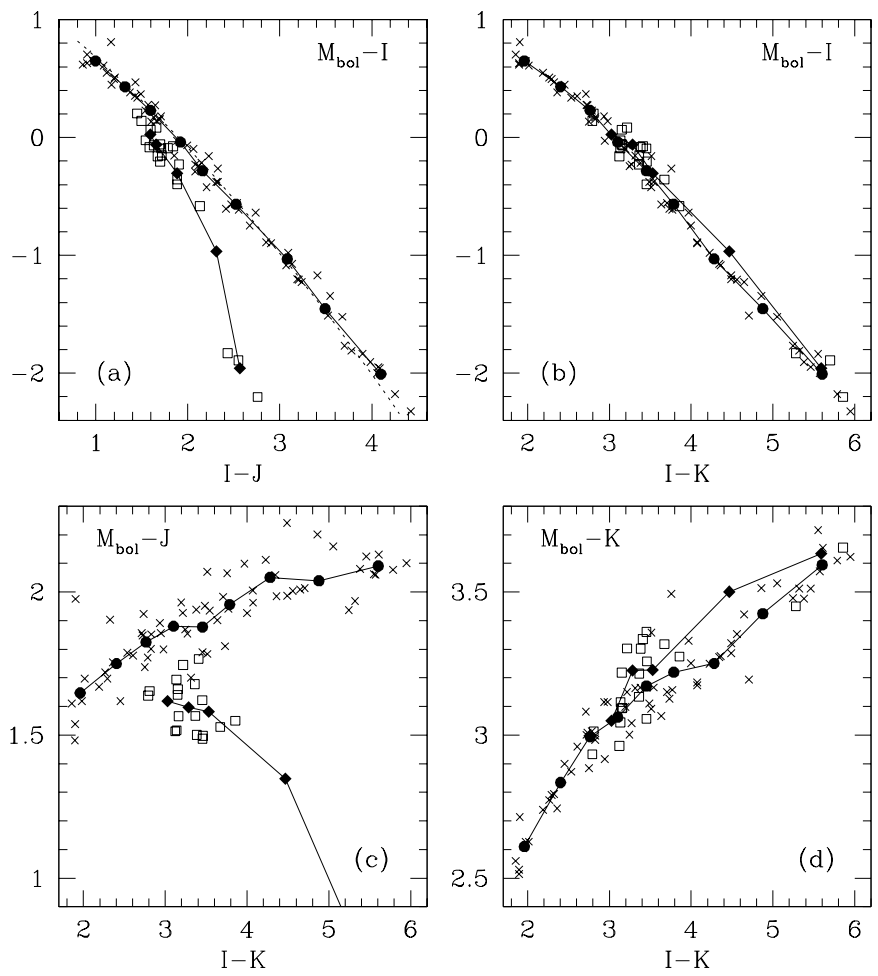

Fig. 10. Bolometric corrections, obtained from the LW2000 data and the mean spectra, with the extrapolation method described in the text. Crosses and open squares: individual stellar spectra for O-rich and Crich LPVs; solid symbols: sequences of averaged spectra. Dotted line in a) values obtained by Alvarez et al. (2000).

makes it impossible to test directly whether the extrapolation is still valid for the coolest carbon star bin, but the near-IR similarity between RLep and the ISO target S Cep (see Goebel et al. 1978 and LW2000) suggests that the linear extrapolation method doesn't underestimate the $L_{\text {bol }}$ by more than $30 \%$ even in that case.

Figure 10 shows that the bolometric corrections $M_{\mathrm{bol}}-I$ and $M_{\text {bol }}-J$ for carbon stars lie below those of oxygen rich stars. The same would be true for $M_{\mathrm{bol}}-H$. The explanation lies in the location of the main molecular bands. In O-rich stars, water bands tend to lie at the edge of the standard photometric windows, while in carbon stars the $\mathrm{C}_{2}$ and $\mathrm{CN}$ bands reduce the flux density right through these windows. In comparison, molecular absorption is relatively small in the $K$ window for both chemical classes.

The bolometric corrections above do not apply to AGB stars in opaque dust shells. Applying reddening as suggested in Sect. 3.3 automatically accounts for the reduced optical and near-IR fluxes of these objects. The determination of bolometric corrections is thus directly dependent on the rapidly evolving circumstellar optical depth.

\subsection{Temperature scales}

The second step required in the assignment of a spectrum to a particular stage along a stellar evolution track is the determination of an effective temperature $\left(T_{\text {eff }}\right)$ scale for the available spectra. A straightforward way of doing this is to rely on theoretical or semi-empirical scales (e.g. angular diameter measurements). Although we provide relations of that kind here, we emphasize that with current stellar models all attempts to relate temperatures assigned this way with the $T_{\text {eff }}$ of a particular set of stellar evolution tracks are uncertain. More arguments to this important point are given in Sect. 5.1.

Bessell et al. (1989b) provide temperature scales based on $(I-K)$. The scales are metallicity-dependent. We adopt solar metallicity here and refer to Sect. 6 for a discussion of metallicity effects. Synthetic spectra for static and pulsating AGB stars have been kindly made available to us by M. Scholz. The static models and their colours are described in Bessell et al. (1989b, 1991), the pulsating ones in Bessell et al. (1996; their E, Z and D series) and Hofmann et al. (1998; their L, M, P and $\mathrm{Q}$ series). A detailed comparison between the models and the data lies far beyond the scope of this paper. Although many observed tendencies are reproduced by the models at least in a statistical sense, the fits to individual spectra are not perfect, and from our preliminary analysis we estimate that they do not provide a more reliable absolute effective temperature than, for instance, a colour such as $(I-K)$.

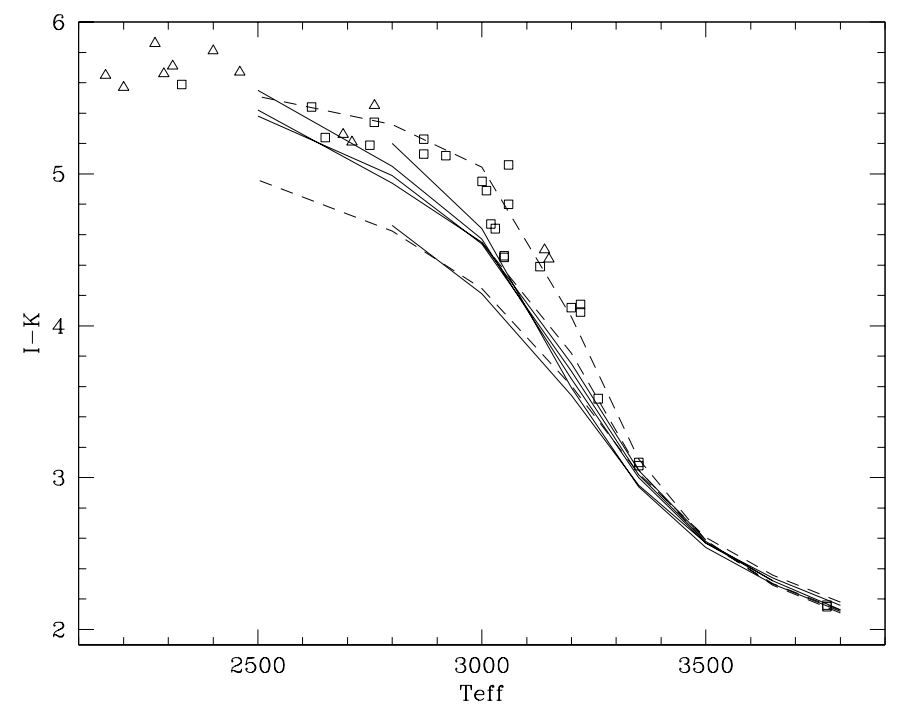

Fig. 11. Effective temperature scales based on synthetic spectra. The lines follow static model series. Dashed (Bessell et al. 1989b): $1 M_{\odot}$ sequences with, from top to bottom $\left(10^{4} L_{\odot} ; Z_{\odot}\right),\left(2 \times 10^{3} L_{\odot} ; Z_{\odot}\right)$, $\left(10^{4} L_{\odot} ; Z_{\odot} / 2\right)$. Solid (Bessell et al. 1991): the bundle of 4 similar lines follow the AGB locus at $Z_{\odot}$ for $1,1.5,2.5$ and $5 M_{\odot}$, and the bluer line is for $1 M_{\odot}$ at $Z_{\odot} / 2$. Symbols (Bessell et al. 1996; Hofmann et al. 1998): dynamical model stars at phase 0.5 (triangles) or 0.8 or 0. (squares).

Figure 11 shows the relation between $(I-K)$ and the effective temperature for solar metallicity models and for two series of static models at subsolar metallicity. The effective temperature is a theoretical quantity, defined using Stefan's law and the radius at which the Rosseland mean optical depth of the star reaches unity. While each of the dashed lines is a sequence of models at constant mass $\left(1 M_{\odot}\right)$ and luminosity, the solid lines 
Table 3. Properties of the averaged spectra.

\begin{tabular}{ccccc}
\hline \hline \multicolumn{5}{c}{ O-rich spectra } \\
\hline Bin & $I-K$ & $J-K(J-K s)^{a}$ & $B C(I)$ & $T$ \\
$(1)$ & $(2)$ & $(3)$ & $(4)$ & $(5)$ \\
\hline 1 & 1.96 & $0.96(1.01)$ & 0.65 & $3930 \mathrm{~K}$ \\
2 & 2.40 & $1.08(1.13)$ & 0.43 & $3585 \mathrm{~K}$ \\
3 & 2.76 & $1.17(1.22)$ & 0.23 & $3440 \mathrm{~K}$ \\
4 & 3.10 & $1.18(1.23)$ & -0.04 & $3355 \mathrm{~K}$ \\
5 & 3.45 & $1.29(1.34)$ & -0.28 & $3285 \mathrm{~K}$ \\
6 & 3.79 & $1.26(1.31)$ & -0.57 & $3235 \mathrm{~K}$ \\
7 & 4.28 & $1.20(1.24)$ & -1.03 & $3175 \mathrm{~K}$ \\
8 & 4.88 & $1.38(1.43)$ & -1.45 & $3055 \mathrm{~K}$ \\
9 & 5.60 & $1.50(1.55)$ & -2.01 & $2340 \mathrm{~K}$ \\
\hline \multicolumn{5}{c}{$\mathrm{C}-$ rich spectra } \\
\hline Bin & $R-H$ & $J-K(J-K s)^{a}$ & $B C(I)$ & $T$ \\
$(1)$ & $(2)$ & $(3)$ & $(4)$ & $(5)$ \\
\hline 1 & 3.74 & $1.43(1.52)$ & 0.02 & $3200 \mathrm{~K}$ \\
2 & 4.06 & $1.63(1.71)$ & -0.06 & $3000 \mathrm{~K}$ \\
3 & 4.38 & $1.65(1.74)$ & -0.30 & $2800 \mathrm{~K}$ \\
4 & 5.08 & $2.15(2.23)$ & $-0.97:$ & $2400 \mathrm{~K}$ \\
5 & 5.86 & $3.03(3.08)$ & $-1.96:$ & $2000 \mathrm{~K}$ \\
\hline
\end{tabular}

Notes to Table 3:

a $(J-K s)$ is given for the DENIS filter passbands (Epchtein et al. 1997), kindly provided by M. Schultheis.

A colon indicates uncertain values, where the far-IR emission is likely to be underestimated by the adopted extrapolation procedure.

For the interpretation of Col. 5, see Sects. 4.2 and 5.1.

follow the luminosity-temperature relations of AGB evolutionary tracks as parametrized by Bessell et al. (1991). The symbols shown for the pulsating models are instantaneous values, corresponding to a particular cycle and pulsation phase. It is seen that the dynamical models follow a sequence that agrees with the $1 M_{\odot}$, high luminosity (i.e. low gravity) static solar metallicity sequence of Bessell et al. (1989b). This locus provides the temperatures of the averaged empirical spectra listed in Col. 5 of Table 3. A lower effective temperature would be assigned to a given spectrum if the subsolar theoretical scales or the solar ones of Bessell et al. (1991) were used instead. The comparison of the range in $(I-K)$ in Table 3 and along the models in Fig. 11 shows that the sequence of average LPV spectra adequately covers the temperature range required for AGB synthesis.

For carbon stars with effective temperatures above $\sim 2600 \mathrm{~K}$, we adopt the relation between $(R-H)$ and $T_{\text {eff }}$ derived by LLJ2001. The values for the binned spectra of Fig. 7 are given in Table 3. The two coolest bins of the sequence contain spectra of RLep, a Mira-type variable with a period of 427 days. A $T_{\text {eff }}$ of $2058 \pm 180 \mathrm{~K}$ has been obtained for this star from interferometric angular size measurements in October, 1995 (van Belle et al. 1997). The data in our temperature bin 5 were taken between December, 1995 and early March, 1996. The 3 individual spectra are very similar; the comparison of their energy distributions suggests that the minimum temperature was reached in early January, 1996, i.e. only 0.2 cycles after the interferometric measurement. We therefore assign the spectrum of bin 5 an effective temperature of $\sim 2000 \mathrm{~K}$. The $(V-K)-T_{\text {eff }}$ relation for Carbon Miras of van Belle et al. (1997) predicts $(V-K) \simeq 10$ at this temperature, which is in good agreement with our measurement (after extrapolation of the observed spectrum to the short wavelength cut-off of the standard $V$ band filters). Based on this sequence, $T_{\text {eff }} \simeq 2400 \mathrm{~K}$ is appropriate for bin 4 . The range of $(J-K)$ values in the average spectra is in excellent agreement with observations in complete carbon star samples (Frogel \& Elias 1988; Glass et al. 1995; Cioni et al. 2000; Weinberg \& Nikolaev 2001).

\section{Discussion}

\subsection{Temperature bins and temperature calibrations}

In the above sections, we have shown that wide baseline colour temperatures provide the most sensible and practical classification of the LPV spectra. When using these binned spectra to synthesize cluster or galaxy spectra, one assumes that the energy-weighted mean spectrum of an individual LPV (averaged over the $\leq 10^{3}$ days of the LPV pulsation cycle) is similar to the average of many individual stellar spectra obtained from stars of various masses, amplitudes and phases, but with a common colour temperature. As we found no significant correlations, in any of our temperature bins, between the deviations from the mean spectral properties and parameters such as amplitude or period, this is a reasonable choice. More observations will be needed before other options can be considered.

With the above assumption, the effective temperature along a TP-AGB evolution track alone determines which spectrum should be used to represent the corresponding emission (it is assumed that the track also indicates when the use of LPV spectra instead of static giants becomes necessary, and when a star becomes C-rich or dust-enshrouded). In order to avoid misinterpretations of the temperature scales given in Table 3 , it is important to recall how evolutionary TP-AGB tracks and individual TP-AGB stars behave in the HR diagram.

Even a perfectly coeval stellar population produces a broad asymptotic giant branch in the HR diagram and in colourmagnitude diagrams, as a result of the thermal pulses (helium shell flashes) that occur on timescales of $\sim 10^{4}$ yrs and of the long period pulsational variability on timescales of $10^{2}-10^{3}$ days. Figure 12 shows theoretical illustrations of these effects, and Fig. 13 provides an empirical counterpart.

The TP-AGB track in Fig. 12 (left) was computed using socalled "synthetic evolution" techniques (Iben \& Renzini 1983; Groenewegen \& de Jong 1993; Wagenhuber \& Groenewegen 1998; Marigo et al. 1998; Mouhcine \& Lançon 2002). The values of the various model parameters for this particular track are not relevant here. Tiny offsets in many stellar parameters will ensure that thermal pulses do not occur in phase for all stars. Most stars will be found around the quiescent luminosity, and $10-30 \%$ at the significantly lower luminosities that follow thermal pulses (Boothroyd \& Sackmann 1988; Groenewegen \& de Jong 1993; Marigo et al. 1999). The locus of any set of TP-AGB tracks is based on its own particular definition of the $T_{\text {eff }}$ (see Scholz \& Takeda 1987 for a discussion), through rather simple outer boundary conditions for such cool and extended stellar atmospheres. In addition, as already mentioned in Sect. 1, these temperatures are those of static model stars. 


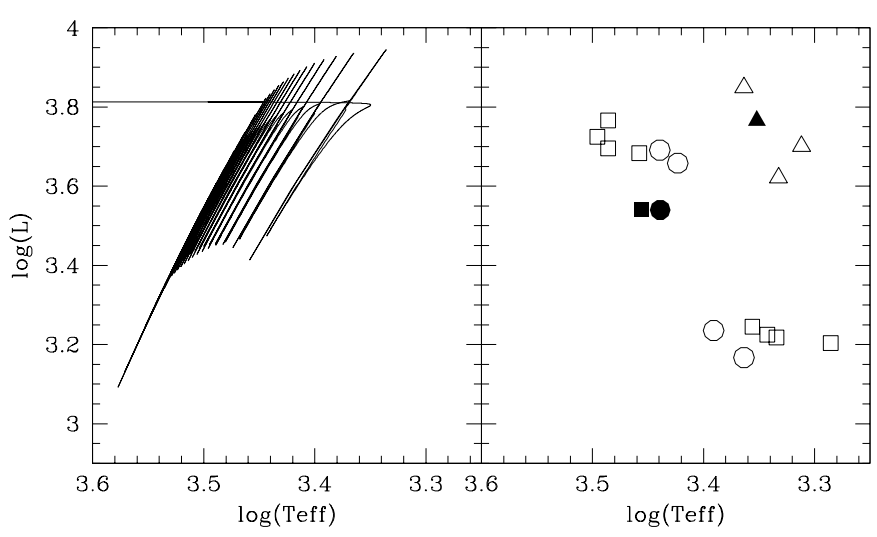

Fig. 12. Left: Synthetic TP-AGB track for the evolution of a star with an initial mass of $2 M_{\odot}$ and a final mass of $0.6 M_{\odot}$. Right: $1 M_{\odot}$ pulsating star models from Hofmann et al. (1998). The P, M and O series are shown respectively as squares, circles and triangles. Properties at maximum and minimum light in several successive cycles are shown (open symbols), together with those of the static parent star (solid).

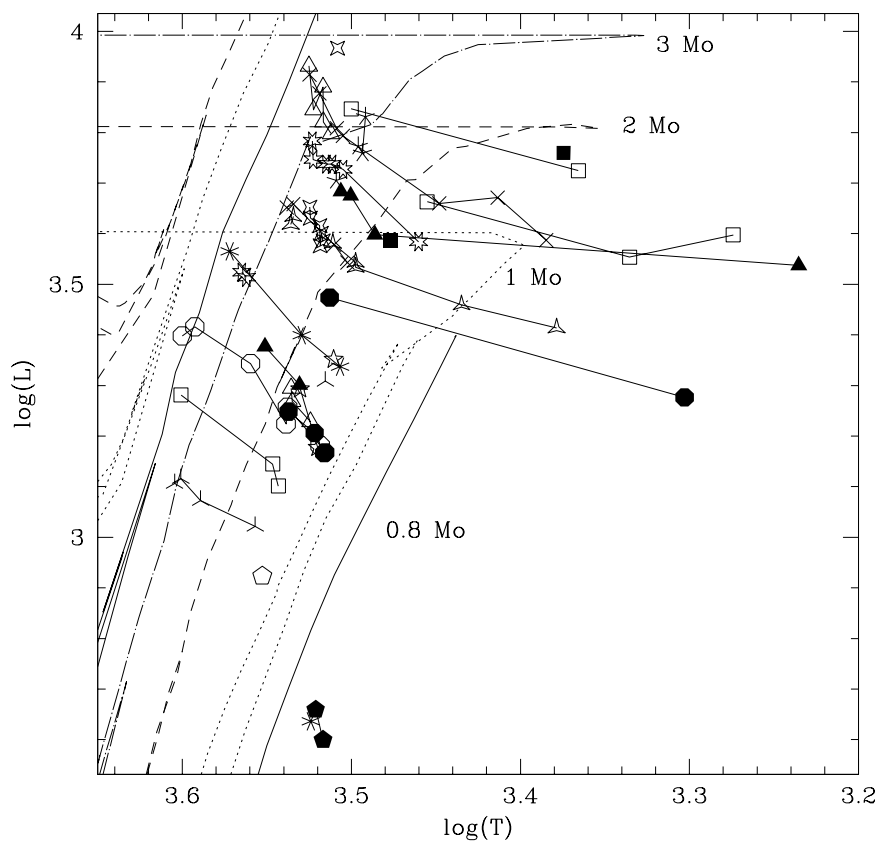

Fig. 13. Tentative location of the LW2000 spectra on the theoretical HR diagram (for stars with known periods). $T_{\text {eff }}$ is derived from $(I-K)$, $\log (L)$ from the period and the instantaneous bolometric correction. Symbols distinguish individual stars; multiple observations at various phases are connected. Smoothed AGB tracks at $Z_{\odot}$ are overlaid (Mouhcine \& Lançon 2002; at $0.8 M_{\odot}$, only the RGB is shown).

The right hand plot of Fig. 12 shows the predicted effect of Mira-type pulsation for the three most recent models described by Hofmann et al. (1998). All models pulsate with periods of the order of 330 days and assume a mass of $1 M_{\odot}$. The two models at relatively lower luminosity (series $\mathrm{P}$ and $\mathrm{M}$ of the authors) are fundamental mode pulsators, while the third one (O series) pulsates in the first overtone. All models behave differently in the HR diagram. Model M shows that pulsation is able to shift a star systematically to larger radii and lower effective temperatures, when compared to its static parent.
This trend is also seen when direct angular diameter measurements of pulsating and static late-type giants are compared (van Belle et al. 1997). However, the amplitude of this effect at a given point of an evolutionary track cannot be predicted reliably because neither the evolution of LPV pulsation modes, nor pulsation amplitudes, nor the actual effect of these quantities on the spectra are understood.

In Fig. 13, the O-rich stars of the LW2000 library have been located tentatively in the theoretical HR diagram, in order to estimate the empirical width of an upper AGB sequence. Each star with a known period has been assigned a $K$ band luminosity $\left(L_{K}\right)$ using the empirically well-defined period $-L_{K}$ relation for Miras by Hughes \& Wood (1990). Note that those stars which pulsate in overtone modes should in fact be assigned higher luminosities (see LW2000 for potential candidates). $L_{K}$ variations have been neglected in the figure. The changes in the bolometric luminosity represent the changes in the instantaneous bolometric corrections measured on the spectra as in Sect. 4.1. The assigned temperatures are derived from $(I-K)$ as in Sect. 4.2. It is seen that pulsation can widen the AGB to $\Delta T=500-1000 \mathrm{~K}$ or $\Delta(I-K)=1-2 \mathrm{mag}$. This range is consistent with the predicted ranges shown in Fig. 12. If pulsation was neglected, such a range of colours could be mistaken for a range of several $M_{\odot}$ in initial stellar masses. Alternatively, it could be attributed to a spread in metallicity. TP-AGB tracks are typically shifted to hotter $T_{\text {eff }}$ by 0.3 dex when going from $Z=0.02$ to $Z=0.008$.

The tracks overlaid in Fig. 13 are smoothed (many population synthesis codes require that thermal pulses be smoothed out, to make interpolations between tracks or the construction of isochrones possible). The smoothed luminosities are the result of energy conservation: smooth tracks run at luminosities slightly lower than the quiescent, pre-flash luminosities, in order to account for the post-flash luminosity dips. More options exist in the smoothing schemes for $T_{\text {eff }}$, as the ideal weighting should use the energy output in the particular wavelength range one intends to make predictions for. The differences between various schemes will however remain small compared to the systematic effects of $T_{\text {eff }}$ definitions or LPV pulsation mentioned above.

In summary, theoretical effective temperatures are not directly comparable to those estimated for individual empirical spectra of LPVs. Acquiring enough data to obtain representative average spectra is only one step, in which the LW2000 sample has allowed significant progress. But until consistent models for the structure and spectrum of pulsating AGB stars exist, the link between the theoretical $T_{\text {eff }}$ scale and any semiempirical one remains uncertain. It must be calibrated a posteriori. Ideal observational targets for the temperature calibrations are massive stellar clusters with known metallicities and ages (from optical spectroscopy), such as found in merger galaxies for instance (Maraston et al. 2001; Mouhcine et al. 2002). The relevant ages lie between $10^{8}$ and $2 \times 10^{9}$ yrs. In individual clusters containing less than $\sim 10^{5}$ stars, the stochastic effects due to the intrinsically small number of bright AGB stars affect the comparison significantly (Lançon \& Mouhcine 2000). 


\subsection{Period and amplitude distributions}

As recalled in Sect. 2, the stars of the LW2000 sample were selected to provide a relatively uniform coverage of the periodamplitude plane. Those used to construct the averages of this paper cover periods of $100 \leq P \leq 450$ and optical amplitudes of $0.8 \leq \delta V \leq 7 \mathrm{mag}$.

Systematic photometric observations of large samples of stars have shown that the majority of the TP-AGB stars are variable (Wood et al. 1999; Alcock et al. 2000; Cioni et al. 2001). However, period and amplitude distributions are expected to depend on the age of the observed population (Vassiliadis \& Wood 1993). Large amplitude pulsation characterizes only a subpopulation of the TP-AGB stars. Here, we discuss whether or not it is appropriate to use the average LPV spectra of this paper for all the TP-AGB stars of a synthetic population.

Period distributions in the Large Magellanic Cloud (LMC) or in the Galaxy extend from less than 20 days to more than $10^{3}$ days. The range of periods of our LPV sample corresponds to those of optically visible Miras and of relatively long period, high luminosity semi-regular stars, as seen in Fig. 1 of Wood (2000). Both in the LMC and in the Galactic Bulge, semi-regular variables with periods shorter than 100 days outnumber Miras (Wood et al. 1999; Alard et al. 2001; Alcock et al. 2000). The mean period and the mean amplitude of our sample are thus larger than the mean period of optically visible TP-AGB stars in populations that have been forming stars for a long time (i.e. since the epoch of galaxy formation).

What is relevant to the synthesis of integrated galaxy or cluster spectra is how the period and amplitude distribution of the available stellar sample compares with the distribution for those particular TP-AGB stars that contribute most strongly to the light (in the near-IR, i.e. the only spectral range of the LW2000 data where the TP-AGB contribution is significant). Population synthesis models have shown that these are stars with initial masses between about 1.8 and $3 M_{\odot}$ (Mouhcine \& Lançon 2002; see also Renzini 1992; Girardi \& Bertelli 1998; Maraston 1998). They reach the TP-AGB with ages of about 0.1 to $1.3 \mathrm{Gyr}$ and spend of the order of $10^{6} \mathrm{yrs}$ in that phase (exact ranges are model and metallicity dependent). In older populations, the stars of the red giant branch are so numerous that they provide most of the near-IR light. Which spectra are used for the remaining insignificant TP-AGB stars stops being critical then.

What do we know about the pulsational properties of stars with initial masses between 1.8 and $3 M_{\odot}$ ? A global picture has emerged from joint studies of empirical period-luminosity distributions and theoretical pulsation models (Fox \& Wood 1982; Xiong et al. 1998; Vassiliadis \& Wood 1993; Wagenhuber \& Tuchman 1996). In the period-luminosity diagram, a single evolutionary track runs "stepwise" accross pulsation mode sequences, from short to long periods, with a slowly increasing luminosity (apart from the temporary changes due to the thermal pulses): a star becomes unstable to one of the overtone pulsation modes, follows that period-luminosity sequence until it switches to a lower overtone, and so on. The large mass loss associated with Mira-type pulsation finally makes the star loose its envelope. Stars with more massive initial masses arrive in the unstable strips of the period-luminosity diagram with larger luminosities. This is supported by the observation that Milky Way Miras with very long periods are associated with the thin disk, while shorter period Miras are seen in the thick disk and in globular clusters (Feast 1963; Habing 1995). The stars with masses between 1.8 and $3 M_{\odot}$ are thus expected to pulsate with larger mean periods than a population in which most stars are old.

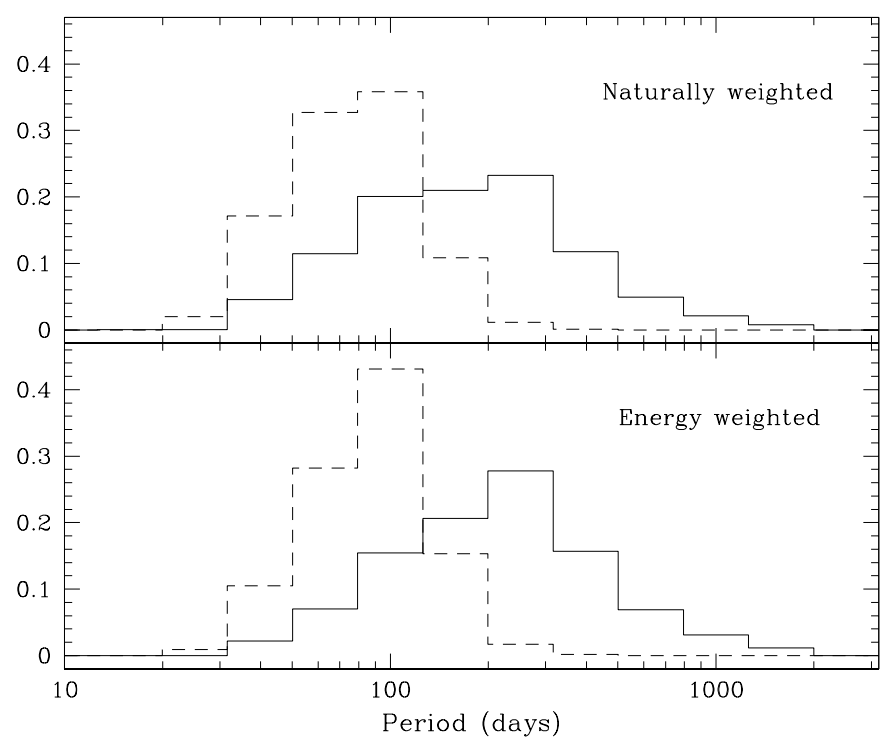

Fig. 14. Predicted period distributions for stars with initial masses around $2 M_{\odot}\left(Z=Z_{\odot}\right)$. Fundamental mode pulsation is assumed for the solid line, first overtone for the dashed line. Each distribution is normalized individually to a sum of one.

Figure 14 shows predicted period distributions for a stars with an initial mass of $2 M_{\odot}$, based on the TP-AGB tracks of Mouhcine \& Lançon (2002), combined with the period-massradius relations of Wood (1990) for fundamental mode and of Barthès \& Luri (2001) for the first overtone. Systematic effects of the initial mass are small between 1.8 and $3 M_{\odot}$. The plotted distribution is representative of the initial masses most relevant to observable integrated properties of stellar populations. The upper graph is a number distribution, the lower gives the relative contributions of stars with various periods to the luminosity. The relative number of fundamental mode and overtone pulsators is very uncertain. In the LMC period-luminosity diagram (Wood et al. 1999), about $1 / 3$ of the LPVs in the relevant, high luminosity range are assigned fundamental mode (unfortunately the LMC scarcely populates the upper part of the diagram, due to the combined effects of the stellar initial mass function and the star formation history). Figure 14 shows that the range of periods available to us for the construction of average spectra is indeed very appropriate for the fundamental mode pulsators. A similar conclusion is reached when other tracks in the literature are used (Vassiliadis \& Wood 1993; Wagenhuber \& Tuchman 1996; Gautschy 1999).

The periods of our spectroscopic sample are however larger than those of the overtone pulsators. According to the data of Wood et al. (1999), the overtone pulsators are essentially 
all semi-regular variables, with small amplitudes compared to those of the Mira-like fundamental mode pulsators (semiregular LPVs are found on the fundamental mode sequence, but at lower luminosities and shorter periods than those relevant here, as seen in the light curves given in Wood et al. 1999). Many of the overtone pulsators should be well represented with spectra of static giants. Thus, the relative importance of the LPVs in synthetic populations can be varied by modifying the blue edge of the instability strip.

Current pulsation theory does not put strong constraints on this edge and on the transition from overtone to fundamental mode pulsation. In order to evaluate the effects of such adjustments, we have compared synthetic spectra of intermediate age populations computed with two assumptions: (i) our average LPV spectra are appropriate for all TP-AGB stars or (ii) the LPV spectra only apply in the Mira instability strip. For (ii), the blue edge of the Mira instability strip was projected from the empirical period-luminosity plane into the HR diagram assuming fundamental mode pulsation models (Wood 1990), and static giant stars were used up to that point. The differences were much smaller than the uncertainties currently associated with, for instance, the effective temperature scales of the spectra (changes were smaller than $3 \%$ in all the quantities we measured).

In summary, the simplifying assumption that the average LPV spectra of this paper apply to all TP-AGB stars is reasonable, when the aim is the synthesis of integrated spectra of stellar populations. Better theoretical predictions for the onset and evolution of period and amplitude of TP-AGB stars with various initial masses will be needed to reduce the uncertainties inherent to this assumption. In the study of resolved populations that include AGB stars with low initial masses, it is likely that the spectra of static giant stars should be used for some as yet poorly defined first part of the TP-AGB.

\subsection{Hydrogen recombination lines}

The only conspicuous near-IR emission line seen in LPVs is Paschen $\beta$, at $1.28 \mu \mathrm{m}$. Emission equivalent widths of up to $6 \AA$ are observed in individual spectra of the LW2000 sample. $\mathrm{Pa} \beta$ is seen in most of the averaged O-rich spectra of Sect. 3. The emission equivalent width is of the order of $1 \AA$ in 6 of the 9 temperature bins. None of the $C$ star spectra of LW2000 shows significant Hydrogen recombination lines. This is mostly due to molecular line crowding, which is more severe in C-rich spectra around the wavelengths of the hydrogen lines. In the maximum light spectrum of the S/C type star $\mathrm{BHCru}, \mathrm{Pa} \beta$ is detected with an equivalent width of the order of $1.5 \AA$ (the resolution of the data defines the pseudocontinuum). Paschen $\beta$ is not seen in the averaged $C$ star spectra of Sect. 3.2.

According to the evolutionary tracks and population synthesis calculations of Mouhcine \& Lançon (2002), TP-AGB stars contribute less than $40 \%$ of the $J$ band light in coeval populations of intermediate ages. If the $\mathrm{Pa} \beta$ equivalent widths of the average LPV spectra are representative of the TP-AGB, those of integrated spectra of stellar populations will not exceed fractions of an Angström. This is small compared to the effect of any significant subpopulation of young ionizing stars (Leitherer et al. 1999).

\section{A recipe for spectral synthesis at various metallicities}

When metallicity is varied, several effects add up to produce differences in the predicted contributions of upper AGB stars to the integrated light of a stellar population. First, the relation between effective temperature and spectrophotometric properties changes. For cool stars, this is due to the importance of molecular opacities. An illustration can be found, e.g., in Fig. 10 of Hauschildt et al. (1999), where static giant model spectra for $[\mathrm{M} / \mathrm{H}]=0$ and -0.3 are compared. When working at lower metallicity, a bluer spectrum with weaker molecular bands must be associated with each given point of the theoretical HR diagram. Second, the AGB evolutionary tracks themselves are globally shifted to lower effective temperatures. This effect acts in the same direction as the first one: signatures of cooler AGB stars will be more pronounced at higher metallicities. The third effect is the metallicity-dependence of the mass loss, of the AGB lifetimes and of the efficiency of the production of carbon stars. Discussions of these latter aspects can be found, e.g., in Groenewegen \& de Jong (1993), Marigo et al. (1998), Mouhcine \& Lançon (2002).

The metallicites of the LW2000 spectra are not known. As the observed stars are located in the solar neighbourhood, they are expected to be representative of stars in approximately solar metallicity environments. The range of ages (or initial masses) in the data is likely to correspond to a range of metallicities, as determined by the chemical evolution of our neighbourhood. A contamination of the sample by Population II stars is also likely (LW2000). The calibrations of Ramírez et al. (2000) and Frogel et al. (2001), that relate $[\mathrm{Fe} / \mathrm{H}]$ to the equivalent widths of near-IR absorption features and colours based on Milky Way cluster red giant branch stars, would lead to dispersed, on average slightly subsolar metallicities. However, the calibration samples don't include LPVs, and the observed variability of the relevant spectral signatures in the LW2000 sample makes the results uncertain. The LW2000 have also been compared to the synthetic red giant spectra of Bessell et al. (1989a) and of Hauschildt et al. (1999). Both sets provide decent (though far from perfect) agreement with the observed giant star spectra and at least some of the LPV spectra. In the models of Hauschildt et al. (1999), the effect of moving from $[\mathrm{M} / \mathrm{H}]=0$ to -0.3 is so strong at low temperatures that the low metallicity model spectra are very different from any observed one we have seen, in the LW2000 data or in the literature. With these models, one would conclude that all the LW2000 stars have quasi-solar metallicities, but our impression is that the effect of $Z$ in the models is too strong. In the model set of Bessell et al. (1989a) moving from $\log \left(Z / Z_{\odot}\right)=0$ to -1 has a relatively small effect on the spectra (at a given $T_{\text {eff }}$, mass and surface gravity), indicating that the observed spectra may be appropriate over that range of metallicities. The comparison between properties of stars in the Galactic Bulge and in the Field supports a modest evolution of intrinsic spectral signatures over a useful range of metallicites (Frogel et al. 1984). 
In view of this situation, we suggest to restrict the inclusion of metallicity effects in spectral population synthesis models to those that are intrinsic to stellar evolutionary tracks, and to the use of a metallicity dependent $T_{\text {eff }}$ scale for the mean $\mathrm{O}$-spectra. For instance, one may use the relations between $(I-K)$ and $T_{\text {eff }}$ given for various metallicities by Bessell et al. (1991). The sensitivity of carbon star spectra to metallicity is small. Gautschy (2001) has computed model spectra for hydrostatic spherical carbon stars between $\log \left(Z / Z_{\odot}\right)=0$ and -0.3 . The models show changes in $Z$ have small effects compared to changes in the effective temperature, the $\mathrm{C} / \mathrm{O}$ ratio or the ${ }^{12} \mathrm{C} /{ }^{13} \mathrm{C}$ ratio. The latter are determined by the current evolutionary status of a star, at any initial metallicity. For carbon stars, we suggest to use the average spectra of this paper with the same effective temperature scale at all $Z$.

\section{Summary and conclusions}

This paper provides convenient and representative data for stars on the upper asymptotic giant branch, to be used in the studies of cool stellar populations of galaxies.

We have used the data of LW2000 to construct sequences of average spectra for O-rich and C-rich LPVs. The O-rich sequence is based on $(I-K)$, which is taken as a first order effective temperature indicator. Despite the large dispersion between $(I-K)$ (or other temperature indicators involving optical data) and near-IR properties, a regular behaviour is observed along the average sequence. This would not have been the case if sorting had been based on near-IR indices. For C-rich stars, both a temperature sequence and a sequence based on the $\mathrm{C} / \mathrm{O}$ ratio are presented. For stars with thick dust envelopes $(\mathrm{OH} / \mathrm{IR}$ stars and their carbon rich equivalent), the use of reddened versions of the above data is suggested.

In studies of stellar populations, the average spectra will be used in connection with stellar evolution tracks or isochrones. The latter are expected to provide the distribution of stars in the HR diagram, but also the onset of LPV pulsation (i.e. when to use the average LPV spectra instead of static giant spectra), the transition between O-rich and C-rich atmospheres, and the transition from an optically visible object to a dust-enshrouded far-IR source. As both the effective temperatures provided by stellar evolution tracks for the thermally pulsing AGB and the temperatures estimated for the average spectra are (independently) model-dependent and uncertain, we suggest caution when using the values indicated in this paper. The effects of reasonable changes in the temperature scales have been illustrated by Lançon et al. (1999). We recommend an a posteriori calibration of the relative temperature scales of the spectra and the evolutionary tracks, based on observed properties of intermediate age star clusters of known ages and metallicities.

Using only one parameter, the effective temperature, to characterize LPV spectra is clearly a first order approximation (even if this particular choice of a parameter is arguably the most appropriate one). Pulsation period and amplitude as well as the phase in the pulsation cycle are expected to produce systematic effects, although neither the LW2000 sample nor the currently available stellar models are sufficient to pin those down. The period and amplitude distributions in the
LW2000 data used here are biased towards high values, when compared to the distribution of LPVs (including Miras and semi-regular variables) found in star counts in the Milky Way or the Magellanic Clouds. As only TP-AGB stars with relatively high main sequence masses will ever contribute much to the integrated light of stellar populations, the problem is not as severe there. In the practice of population synthesis calculations, the errors resulting from this period and amplitude distribution cannot be disentangled from errors in the assumed onset instants of semi-regular and Mira-type pulsation.

Acknowledgements. We thank M. Scholz for providing his model spectra in digital form, D. Barthès for pointing out a correction to standard overtone period-luminosity relations and providing his results in advance of publication, F. Kerschbaum and B. Aringer for their help with the ISO spectra they made available to us, and P. Wood for his suggestions and support throughout this work.

\section{References}

Alard, C., the ISOGAL Collaboration, \& the MACHO Collaboration 2001, ApJ, 552, 289

Alcock, C., \& the MACHO Collaboration 2000, AJ, 119 , 2194

Alvarez, R., \& Plez, B. 1998, A\&A, 330, 1109

Alvarez, R., Lançon, A., Plez, B., \& Wood, P. R. 2000, A\&A, 353, 322

Aoki, W., Tsuji, T., \& Ohnaka, K. 1999, in Asymptotic Giant Branch Stars, ed. T. Le Bertre, A. Lèbre, \& C. Waelkens (San Francisco: ASP), IAU Symp., 191, 175

Aringer, B., Kerschbaum, F., Hron, J., et al. 1999, in IAU Symp. 191, Asymptotic Giant Branch Stars, ed. T. Le Bertre, A. Lèbre, \& C. Waelkens (San Francisco: ASP), 169

Barthès, D., \& Luri, X. 2001, A\&A, 365, 519

Bessell, M. S. 1990, PASP, 102, 1181

Bessell, M. S., \& Brett, J. M. 1988, PASP, 100, 1134

Bessell, M. S., Brett, J. M., Scholz, M., \& Wood P. R. 1989a, A\&A, 213, 209

Bessell, M. S., Brett, J. M., Scholz, M., \& Wood, P. R. 1989b, A\&AS, 77,1

Bessell, M. S., Brett, J. M., Scholz, M., \& Wood, P. R. 1991, A\&AS, 89, 335

Bessell, M. S., Scholz, M., \& Wood, P. R. 1996, A\&A, 307, 481

Bessell, M. S., Castelli, F., \& Plez, B. 1998, A\&A, 333, 231

Boothroyd, A. I., \& Sackmann, I.-J. 1988, ApJ, 328, 632

Bressan, A., Granato, G. L., \& Silva, L. 1998, A\&A, 332, 135

Cardelli, J. A., Clayton, G. C., \& Mathis, J. S. 1989, ApJ, 345, 245

Cioni, M.-R. L., van der Marel, R. P., Loup, C., \& Habing, H. J. 2000, A\&A, 359, 601

Cioni, M.-R. L., Marquette, J.-B., Loup, C., et al. 2001, A\&A, 377, 945

Epchtein, N., \& the DENIS Collaboration 1997, The Messenger, 87, 27

Feast, M. W. 1963, MNRAS, 125, 367

Feast, M. W., Glass, I. S., Whitelock, P. A., \& Catchpole, R. M. 1989, MNRAS, 241, 375

Ferraro, F. R., Fusi Pecci, F., Testa, V., et al. 1995, MNRAS, 272, 391

Fluks, M. A., Plez, B., Thé, P. S., et al. 1994, A\&AS, 105, 311

Forestini, M., \& Charbonnel, C. 1997, A\&AS, 123, 241

Fox, M. W., \& Wood, P. R. 1982, ApJ, 259, 198

Frogel, J. A., \& Elias, J. H. 1988, ApJ, 324, 823

Frogel, J. A., Whitford, A. E., \& Rich, R. M. 1984, AJ, 89, 1536 
Frogel, J. A., Mould, J., \& Blanco, V. M. 1990, ApJ, 352, 96

Frogel, J. A., Stephens, A. W., Ramírez, S., \& DePoy, D. L. 2001, AJ, submitted [astro-ph/0101275]

Gautschy, A. 1999, A\&A, 349, 209

Gautschy, R. (previously R. Loidl) 2001, Ph.D. Thesis, University of Vienna, Austria, Feb. 2, 2001

Girardi, L., \& Bertelli, G. 1998, MNRAS, 300, 533

Glass, E. S., Whitelock, P. A., Catchpole, R. M., \& Feast, M. W. 1995, MNRAS, 273, 383

Goebel, J. H., Bregman, J. D., Strecker, D. W., Witteborn, F. C., \& Erickson, E. F. 1978, ApJ, 222, L129

Groenewegen, M. A. T., \& de Jong, T. 1993, A\&A, 267, 410

Habing, H. J. 1995, Mem. Soc. Astr. It., 66, 627

Hauschildt, P. H., Allard, F., Ferguson, J., Baron, E., \& Alexander, D. R. 1999, ApJ, 525, 871

Hofmann, K.-H., Scholz, M., \& Wood, P. R. 1998, A\&A, 339, 846

Hughes, S. M. G., \& Wood, P. R. 1990, AJ, 99, 784

Iben, I. Jr, \& Renzini, A. 1983, ARA\&A, 21, 271

Jørgensen, U. G., Hron, J., \& Loidl, R. 2000, A\&A, 356, 253

Jørgensen, U. G., Jensen, P., Sørensen, G. O., \& Aringer, B. 2001, A\&A, 372, 249

Kholopov, P. N., Samus, N. N., Frolov, M. S., et al. 1985, General Catalogue of Variable Stars (Nauka Publishing House, Moscow)

Kleinmann, S. G., \& Hall, D. N. B. 1986, ApJS, 62, 501

Lançon, A. 1998, in Asymptotic Giant Branch Stars, ed. T. Le Bertre, A. Lèbre \& C. Waelkens (San Francisco: ASP), IAU Symp., 191, 579

Lançon, A., \& Rocca-Volmerange, B. 1992, A\&AS, 96, 593

Lançon, A., \& Mouhcine, M. 2000, in Massive Stellar Clusters, ed. A. Lançon, \& C. M. Boily, ASP Conf. Ser., 211, 43

Lançon, A., \& Wood, P. R. 2000 (LW2000), A\&AS, 146, 217

Lançon, A., Mouhcine, M., Fioc, M., \& Silva, D. 1999, A\&A, 344, L21

Leitherer, C., Schaerer, D., Goldader, J. D., et al. 1999, ApJS, 123, 3, A\&AS, 130, 65

Lloyd Evans, T. 1983, MNRAS, 204, 961

Loidl, R., Lançon, A., \& Jørgensen, U. G. 2001, A\&A, 371, 1065 (LLJ2001)

Maraston, C. 1998, MNRAS, 300, 872
Maraston, C., Kissler-Patig, M., Brodie, J. P., Barmby, P., \& Huchra, J. P. 2001, A\&A, 370, 176

Marigo, P. 2001, A\&A, 370, 194

Marigo, P., Bressan, A., \& Chiosi, C. 1998, A\&A, 331, 564

Marigo, P., Girardi, L., \& Bressan, A. 1999, A\&A, 344, 123

Matsuura, M., Yamamura, I., Murakami, H., \& Onaka, T. 2001, in Post-AGB Objects as a Phase of Stellar Evolution, ed. R. Szczerba, \& S. K. Górny (Dordrecht: Kluwer), Astroph. \& Sp. Sc. Lib., 265, 433

Mouhcine, M., \& Lançon, A. 2002, A\&A, 393, 149 (Paper I)

Mouhcine, M., Lançon, A., Leitherer, C., Silva, D., \& Groenewegen, M. A. T., 2002, A\&A, 393, 101

Persson, S. E., Aaronson, M., Cohen, J. G., Frogel, J. A., \& Matthews, K. 1983, ApJ, 266, 105

Pickles, A. J. 1998, PASP, 110, 863

Ramírez, S. V., Stephens, A. W., Frogel, J. A., \& DePoy, D. L. 2000, AJ, 120, 833

Renzini, A. 1992, in The Stellar Populations of Galaxies, ed. B. Barbuy, \& A. Renzini (Dordrecht: Kluwer), IAU Symp., 149, 325

Renzini, A., \& Voli, M. 1981, A\&A, 94, 175

Renzini, A., \& Buzzoni, A. 1986, in Spectral Evolution of Galaxies, ed. C. Chiosi, \& A. Renzini (Dordrecht: Reidel), 195 galaxies

Scholz, M., \& Takeda, Y. 1987, A\&A, 186, 200 (erratum 1988, A\&A, 196, 342)

Spinrad, H., \& Wing, R. F. 1969, ARA\&A, 7, 249

Terndrup, D. M., Frogel, J. A., \& Whitford, A. E. 1991, ApJ, 378, 742

van Belle, G. T., Dyck, H. M., Thompson, R. R., Benson, J. A., \& Kannappan, S. J. 1997, AJ, 114, 2150

Vassiliadis, E., \& Wood, P. R. 1993, ApJ, 413, 641

Wagenhuber, J., \& Groenewegen, M. A. T. 1998, A\&A, 340, 183

Wagenhuber, J., \& Tuchman, Y. 1996, A\&A, 311, 509

Weinberg, M. D., \& Nikolaev, S. 2001, ApJ, 548, 712

Wood, P. R. 1990, in From Miras to Planetary Nebulae: Which Path for Stellar Evolution?, ed. M.-O. Mennessier, \& A. Omont (Gifsur-Yvette: Éditions Frontières), 67

Wood, P. R. 2000, PASA, 17, 18

Wood P. R., and the MACHO Collaboration 1999, in IAU Symp. 191, Asymptotic Giant Branch Stars, ed. T. Le Bertre, A. Lèbre, \& C. Waelkens (San Francisco: ASP), 151

Xiong, D. R., Deng, L., \& Cheng, Q. L. 1998, ApJ, 499, 355 